REPRESENTATION THEORY

An Electronic Journal of the American Mathematical Society

Volume 13, Pages 427-459 (September 24, 2009)

S 1088-4165(09)00351-3

\title{
THE WEIL-STEINBERG CHARACTER OF FINITE CLASSICAL GROUPS
}

\author{
G. HISS AND A. ZALESSKI, \\ WITH AN APPENDIX BY OLIVIER BRUNAT
}

\begin{abstract}
We compute the irreducible constitutents of the product of the Weil character and the Steinberg character in those finite classical groups for which a Weil character is defined, namely the symplectic, unitary and general linear groups. It turns out that this product is multiplicity free for the symplectic and general unitary groups, but not for the general linear groups.

As an application we show that the restriction of the Steinberg character of such a group to the subgroup stabilizing a vector in the natural module is multiplicity free. The proof of this result for the unitary groups uses an observation of Brunat, published as an appendix to our paper.

As our "Weil character" for the symplectic groups in even characteristic we use the 2-modular Brauer character of the generalized spinor representation. Its product with the Steinberg character is the Brauer character of a projective module. We also determine its indecomposable direct summands.
\end{abstract}

\section{INTRODUCTION}

The Steinberg character of a finite group of Lie type plays a prominent role in its representation theory. During the last two decades numerous papers have proved the significance of the Weil characters, although these are defined only for classical groups.

In this paper we study the product of the Weil characters with the Steinberg character. For brevity we refer to such a procuct as the Weil-Steinberg character. Our main result claims that the decomposition of the Weil-Steinberg character as the sum of ordinary irreducible characters is multiplicity free for the symplectic and the unitary groups. In fact, we provide much information about these irreducible constituents. One of the striking consequences is that the Weil-Steinberg character is very similar to the Gelfand-Graev character, in the sense that the majority of the irreducible constituents of the latter occur in the former and conversely.

Thus the Weil-Steinberg character can be viewed as a kind of deformation of the Gelfand-Graev character. (However, we do not think that the method used for proving that the Gelfand-Graev character is multiplicity free can be used for proving our result for the Weil-Steinberg character.) As the Gelfand-Graev character plays a fundamental role in the representation theory of groups of Lie type, one could expect that the Weil-Steinberg character will also appear significant.

Received by the editors September 26, 2007 and, in revised from, June 14, 2008.

2000 Mathematics Subject Classification. Primary 20G40, 20C33.

Key words and phrases. Weil character, Steinberg character, classical groups.

(C)2009 American Mathematical Society Reverts to public domain 28 years from publication 
At the moment we have two applications of our results. The first one is on the restriction of the Steinberg character to the stabilizer of a vector of the natural module. We deduce that this restriction is multiplicity free. In addition, we provide significant information on its irreducible constituents. We hope that this will stimulate progress in the long-standing open problem of computing the restriction of an arbitrary representation to the parabolic subgroup which is the stabilizer of an isotropic line of the natural module. Note that our proof used substantially the ideas of the work of Jianbei An and the first author [1, who obtained this result for small-dimensional symplectic groups.

The second line of application of our method could be to compute decomposition numbers. The Weil-Steinberg character is the character of the lift of a projective module in the defining characterisitic, which is the direct sum of some principal indecomposable modules (PIMs for brevity). A straightforward consequence of our results is that each of these PIMs decomposes multiplicity freely as the sum of ordinary irreducible characters, hence certain columns of the decomposition matrix consist of the numbers 1 and 0 only. We do not determine these PIMs here, but there are hints that a number of them are not too small.

Formally, the Weil character cannot be defined for symplectic groups in characteristic 2. However, the Brauer character of a certain module (which we call the generalized spinor module) is an analogue of the Weil character in odd characteristic. Using this analogy, we obtain a similar result for symplectic groups in even characteristic, namely, we show that the product of the generalized spinor Brauer character with the Steinberg character is multiplicity free when decomposed as the sum of ordinary irreducible characters. In contrast with the odd characterisitic case, we also decompose this product as a direct sum of PIMs.

Before we state our main result, we need to specify precisely what we mean by the Weil character in each case.

Definition 1.1. Let $n>1$ be an integer, $q$ a power of the prime $p$ and let $G=G_{n}(q)$ denote one of the following groups: $\operatorname{Sp}(2 n, q), U(2 n, q), U(2 n+1, q)$, or $\mathrm{GL}(n, q)$.

(1) If $G=\operatorname{Sp}(2 n, q)$ with $q$ odd, we let $\omega$ denote the character of one of (the two) Weil representations of $G$ as introduced by Gérardin 11 .

(2) If $G=S p(2 n, q)$ with $q$ even, we let $\omega$ denote the class function obtained by extending the Brauer character of the generalized spinor representation $\sigma_{n}$ of $G$ by zeros on all of $G$. (For a precise definition see Subsection 4.2 below.)

(3) If $G$ is a unitary group, we let $\hat{\omega}$ denote the character of the (unique) Weil representation of $G$ as introduced by Gérardin [11, and define $\omega$ by $\omega:=\hat{\omega}$ if $q$ is even, and by $\omega(g):=\operatorname{det}(g)^{(q+1) / 2} \hat{\omega}(g), g \in G$ if $q$ is odd.

(4) If $G=\operatorname{GL}(n, q)$, we let $\hat{\omega}$ denote the permutation character of $G$ on its natural module, and define $\omega$ by $\omega:=\hat{\omega}$ if $q$ is even, and by $\omega(g):=\operatorname{det}(g)^{(q-1) / 2} \hat{\omega}(g), g \in G$ if $q$ is odd.

In each case, $\omega$ is a class function of $G$ of degree $q^{n}$; in fact, $\omega$ is a character of $G$ except in case (2). We are interested in the product $\omega \cdot$ St, where St denotes the Steinberg character of $G$. Since the Steinberg character vanishes on $p$-singular elements, only the values of $\omega$ on $p$-regular, i.e., semisimple elements of $G$ are relevant. (The two Weil characters of a symplectic group in odd characteristic have the same restriction to the set of semisimple elements, so our choice made in case (1) of Definition 1.1 is not effective.) Let $V$ be the natural module for $G$, and let $g \in G$. Write $N(V ; g):=\operatorname{dim} \operatorname{Ker}(g-1)$ for the dimension of the 1-eigenspace of $g$ on $V$. 
Then if $g \in G$ is semisimple, we have $\omega(g)= \pm q^{N(V ; g) / 2}$ if $G$ is a symplectic group, and $\omega(g)= \pm q^{N(V ; g)}$, otherwise. (For the sign in cases (1) and (3) of Definition 1.1 see [11, Corollaries 4.8.1, 4.8.2].)

The product $\omega \cdot$ St is an ordinary character of $G$, even in case (2) of Definition 1.1. Since St is of $p$-defect 0 , its product with any ordinary character or (extended) $p$ modular character as in case (2) is the character of the lift of a projective module of $G$ in characteristic $p$.

We can now formulate the main result of our paper.

Theorem 1.2. Let $q$ be a power of the prime $p$. For a non-negative integer $m$ let $G_{m}(q)$ denote one of the following groups: $\operatorname{Sp}(2 m, q), U(2 m, q), U(2 m+1, q)$, or $\mathrm{GL}(m, q)$ (with the convention that $G_{0}(q)$ is the trivial group).

Fix a positive integer $n>1$, put $G:=G_{n}(q)$, and denote by $V$ the natural module for $G$. Let $P_{m}$ denote the stabilizer in $G$ of a totally isotropic subspace of $V$ of dimension $m$, so that the Levi subgroup of $P_{m}$ equals $\operatorname{GL}(m, q) \times G_{n-m}(q)$ (respectively, $\operatorname{GL}\left(m, q^{2}\right) \times G_{n-m}(q)$ if $G$ is unitary).

Let St denote the character of the Steinberg representation of $G$, and let $\omega$ be the class function introduced in Definition 1.1. Then

$$
\omega \cdot \mathrm{St}=\sum_{m=0}^{n}\left(\operatorname{Infl}_{P_{m}}\left(\mathrm{St}_{m}^{-} \otimes \gamma_{n-m}^{\prime}\right)\right)^{G} .
$$

Here, $\mathrm{St}_{m}^{-}=1^{-} \cdot \mathrm{St}_{m}$, where $\mathrm{St}_{m}$ denotes the Steinberg character of $\mathrm{GL}(m, q)$ (respectively, $\left.\mathrm{GL}\left(m, q^{2}\right)\right)$, and $1^{-}$the unique linear character of this group of order 2 , if $q$ is odd, and the trivial character, otherwise.

Moreover, $\gamma_{n-m}^{\prime}$ is the Gelfand-Graev character of $G_{n-m}(q)=\mathrm{GL}(n-m, q)$ if $G$ is the general linear group. In the other cases, $\gamma_{n-m}^{\prime}$ is a "truncated" GelfandGraev character of $G_{n-m}(q)$ : It is the sum of the regular characters of those Lusztig series which correspond to semisimple elements without eigenvalue $(-1)^{q}$ on $V$.

We are now going to discuss some consequences of the main result.

Corollary 1.3. Let the notation be as in Theorem 1.2 and suppose that $G$ is not the general linear group. Then the character $\omega \cdot \mathrm{St}$ is multiplicity free.

We remark that this statement is not true for the general linear groups.

The above corollary is one of the principal ingredients in the proof of the following result. As indicated at the beginning of the introduction, this also contains the main motivation for our work.

Theorem 1.4. Let $G$ be one of the groups of Theorem 1.2 and let $H^{\prime}$ denote the stabilizer of a vector in the natural module for $G$. Then the restriction of the Steinberg character of $G$ to $H^{\prime}$ is multiplicity free. In particular, the same conclusion holds for the stabilizer $H$ of a line.

We do not know whether the analogous result holds for the orthogonal groups.

The irreducible characters of $H^{\prime}$ and $H$ can be classified and our proof in fact describes all the irreducible constituents of the restriction of St to $H^{\prime}$ or $H$ (see Subsection 7.1). In case $G$ is a general linear group the above result is well known (see, e.g., [5, Chapter 5]) and its proof does not involve the product $\omega \cdot$ St. To prove the result in case $G$ is a unitary group and $H^{\prime}$ is the stabilizer of an anisotropic vector (i.e., $H^{\prime}$ is a unitary group of one degree less), we use, in addition, a nice 
observation by Olivier Brunat (see the appendix): The restriction of the Steinberg character of $G$ to $H^{\prime}$ is the Weil-Steinberg character of $H^{\prime}$.

A result as in Theorem 1.4 is in general not true for other groups of Lie type. An example is provided by the Chevalley group $G_{2}(q)$. This group has two maximal standard parabolic subgroups $P$ and $Q$. Their character tables have been computed in 2 in case $q$ is odd and not a power of 3. Let $q$ be such a prime power and let $G=G_{2}(q)$. Then, in the notation of [2, the restriction of $\mathrm{St}_{G}$ to $P$ contains the irreducible character ${ }_{P} \theta_{2}(0)$ with multiplicity $(q+1) / 2$ (see [2, Table A.4]), and the restriction of $\mathrm{St}_{G}$ to $Q$ has scalar product $q+1$ with the sum ${ }_{Q} \theta_{5}(0)+{ }_{Q} \theta_{6}(0)$ of two irreducible characters (see [2, Table A.7]). So neither is the restriction of $\mathrm{St}_{G}$ to the maximal parabolic subgroups multiplicity free, nor are these multiplicities bounded independently of $q$.

Theorem 1.4 has some interesting consequences for the $\ell$-modular representation theory of $G$ for $\ell \nmid q$. Namely, the multiplicites of the $\ell$-modular constituents of (the reduction modulo $\ell$ ) the Steinberg character of $G$ can be controlled to some extent by the $\ell$-modular decomposition numbers of $H$. An example of such an application to $\operatorname{Sp}(6, q)$ is given in [1, Section 5].

The Steinberg character is of defect 0 in the defining characteristic. In this case, $\omega \cdot$ St is the ordinary character of a projective module $M$. Thus Corollary 1.3 yields PIMs which are multiplicity free as ordinary characters. In the case of the symplectic groups in characteristic 2 we were able to work out the decomposition of $M$ as a direct sum of PIMs. In order to state this result, we need to recall some notions of algebraic group theory. Let $q$ be a power of 2 and let $\mathbf{K}$ denote an algebraic closure of the finite field $\mathbb{F}_{q}$. Let $\mathbf{G}=\operatorname{Sp}(2 n, \mathbf{K})$ be the symplectic group of degree $2 n$ over $\mathbf{K}$. Furthermore, let $F$ be a standard Frobenius map of $\mathbf{G}$, so that $G:=\mathbf{G}^{F}=\operatorname{Sp}(2 n, q)$ is the finite symplectic group of degree $2 n$ over $\mathbb{F}_{q}$ as in Theorem 1.2. If $\nu$ is a dominant weight of $\mathbf{G}$ we denote by $\phi_{\nu}$ the rational irreducible representation of $\mathbf{G}$ corresponding to $\nu$. If $\nu$ is, furthermore, $q$-restricted, we write $\Phi_{\nu}$ for the principal indecomposable character of $G$ corresponding to the irreducible $\mathbb{F}_{q} G$-representation obtained by restricting $\phi_{\nu}$ to $G$.

Theorem 1.5. Let $\lambda_{1}, \ldots, \lambda_{n}$ be the fundamental weights of $\mathbf{G}$ (ordered as in Bourbaki [4]). Let $\nu_{j}=(q-1) \lambda_{1}+\cdots+(q-1) \lambda_{n-1}+j \lambda_{n}$ for $0 \leq j<q$. Then

$$
\omega \cdot \mathrm{St}=\sum_{j=0}^{q-1} \Phi_{\nu_{j}} .
$$

It follows that the decomposition of every $\Phi_{\nu_{j}}$ as the sum of ordinary characters is multiplicity free. We are not able to distribute the ordinary irreducible constituents of $\omega \cdot$ St described in Theorem 1.2 between the projective indecomposable characters determined in Theorem 1.5. (This distribution will depend on the chosen 2-modular system used to define $\omega$ and the $\Phi_{\nu_{j}}$.)

Our approach is based on Deligne-Lusztig theory. In particular, we have to pass to dual groups in some arguments. The Weil characters of the classical groups (where they exist) are closely related to properties of the natural module for the groups. This is already apparent from the values of these characters on semisimple elements as indicated above. Most important for our results, however, is the following property. Consider a decomposition of the natural module into a direct sum of non-degenerate subspaces. The stabilizer of this decomposition is a direct product of classical groups induced on the subspaces, and the Weil character restricts to this 
stabilizer as a product of the Weil characters of these factors. Such stabilizers are in general not compatible with duality of reductive groups. This is the reason why we take some care in Sections 2 and 3 to derive the necessary facts about maximal tori in duality and their actions on the natural modules.

We conclude this introduction with an outline of the paper. In Section 2 we discuss maximal tori in classical groups and a decomposition of the natural module with respect to a given maximal torus. In Section 3 we relate these decompositions for classical groups in duality. Section 4 introduces the Weil representations and their characters and derives their properties needed later on. In Section 5 we prove Theorem 1.2 for the symplectic and unitary groups, as well as Corollary 1.3. The proof of Theorem 1.2 for the general linear groups is given in Section 6. It is different from the proof for the other classical groups. Section 7 is devoted to the applications of our main result, Theorems 1.4 and 1.5 .

\section{TORI IN CLASSICAL GROUPS}

Let $V$ be a finite-dimensional non-degenerate unitary, symplectic or orthogonal space over the finite field $\mathbb{F}_{q}$ with $q$ elements if $V$ is symplectic or orthogonal, and $q^{2}$ elements if $V$ is unitary. We further assume that $\operatorname{dim} V$ is odd if $V$ is orthogonal. In the latter case we let $G$ be the group of isometries of determinant 1, otherwise $G$ is the group of all isometries of $V$. Thus $G$ is one of the groups $U(V), \operatorname{Sp}(V)$, or $\mathrm{SO}(V)$.

In Subsection 2.1 below we describe a decomposition of $V$ relative to a maximal torus $T$ of $G$ and some formal properties of this decomposition needed later on.

The concept of a maximal torus is defined via the algebraic group underlying $G$. We also have to compare such decompositions of the natural module for groups which are dual to each other in the sense of Deligne and Lusztig, with respect to dual maximal tori. In Subsection 2.2 we therefore introduce maximal tori and the corresponding decompositions of $V$ from an algebraic group point of view. This treatment will also give proofs for the statements in 2.1 and allows us to avoid addressing uniqueness questions which arise for small values of $q$.

2.1. The $T$-decomposition of $V$. Let $T$ be a maximal torus in $G$.

We will call an orthogonal direct sum decomposition

$$
V=V_{0} \oplus V_{1} \oplus \cdots \oplus V_{k} \oplus V_{k+1} \oplus \cdots \oplus V_{k+l},
$$

a $T$-decomposition of $V$, if it has the following properties:

2.1.1. The subspaces $V_{i}$ are non-degenerate $T$-submodules for $1 \leq i \leq k+l$, $V_{k+1}, \ldots, V_{k+l}$ are irreducible, and $V_{1}, \ldots, V_{k}$ are reducible and each of these $V_{i}$ is the sum of two irreducible, totally singular $T$-submodules of equal dimension. Moreover, $V_{0}=\{0\}$ in the unitary and symplectic case; otherwise $V_{0}$ is a 1-dimensional subspace spanned by an anisotropic vector, and $T$ acts trivially on $V_{0}$.

2.1.2. For $1 \leq i \leq k+l$, let $G_{i}$ be the subgroup of $G$ fixing $V_{i}$ and acting as the identity on the orthogonal complement of $V_{i}$. Then $G_{i} \cong U\left(V_{i}\right), \operatorname{SO}\left(V_{i}\right)$, or $\operatorname{Sp}\left(V_{i}\right)$, respectively. Let $H$ be the subgroup of $G$ generated by the $G_{i}$. Then $H$ stabilizes all subspaces $V_{1}, \ldots, V_{k+l}$ and we have $H=G_{1} \times \cdots \times G_{k+l}$. Put $T_{i}=T \cap G_{i}$. We then require that $T_{i}$ is a cyclic maximal torus of $G_{i}$ for all $i$ and

$$
T=T_{1} \times \cdots \times T_{k+l} .
$$


2.1.3. Let $\mu_{i}=\operatorname{dim} V_{i}$ in case $V$ is a unitary space. Then $\mu_{i}$ is even for $1 \leq i \leq k$, and odd, otherwise. In the other cases, each $V_{i}$ for $i \geq 1$ has even dimension and we write $\operatorname{dim} V_{i}=2 \mu_{i}$. For $1 \leq i \leq k$ we have $\left|T_{i}\right|=q^{\mu_{i}}-1$, and for $k+1 \leq i \leq k+l$ we have $\left|T_{i}\right|=q^{\mu_{i}}+1$.

We will show below that a $T$-decomposition of $V$ always exists. Of course, the three conditions above are not independent. Clearly, one can always find a decomposition (11) of $V$ satisfying 2.1.1. Also, 2.1.3 follows from 2.1.2, and, in a generic situation, 2.1.2 is implied by 2.1.1 Consider, however, the case $q=2$ and $V$ symplectic of dimension 4 . Then $G=\operatorname{Sp}(4,2)$. Let $T$ be the maximal torus of order 3 which is the Coxeter torus of the split Levi subgroup GL $(2,2)$ of $G$. Thus there is a $T$-decomposition of $V$ with $k=1$ and $l=0$. There also is a decomposition of $V$ into an orthogonal direct sum of two non-degenerate 2-dimensional irreducible $T$-submodules. This decomposition does not satisfy 2.1.2.

Lemma 2.1. If $G$ is unitary or $q>2$, every maximal torus of $G$ induces a unique $T$-decomposition (up to reordering) of $V$.

Otherwise, any $T$-decomposition refines the decomposition $V=V^{T} \oplus\left(V^{T}\right)^{\perp}$, where $V^{T}:=\{v \in V \mid t v=v$ for all $t \in T\}$. More precisely, $V^{T}=V_{0} \oplus V_{1} \oplus \cdots \oplus V_{k^{\prime}}$ for some $k^{\prime} \leq k$. The decomposition $\left(V^{T}\right)^{\perp}=V_{k^{\prime}+1} \oplus \cdots \oplus V_{k+l}$ is unique (up to reordering), whereas the $V_{i}$ in the decomposition $V^{T}=V_{0} \oplus V_{1} \oplus \cdots \oplus V_{k^{\prime}}$ are hyperbolic planes (and so this decomposition is not unique).

Proof. The existence of a $T$-decomposition will be proved in Subsections 2.3 and 2.4 below. Suppose first that $\left|T_{i}\right|>1$. As $T_{i}$ acts non-trivially on $V_{i}$ but trivially on $V_{j}$ for $j \neq i$, it follows that $V_{i}$ and $V_{j}$ are not isomorphic (as $\mathbb{F}_{q^{2}} T$-modules, respectively, $\mathbb{F}_{q} T$-modules). Hence $V_{i}$ is a homogeneous component of $V$ provided it is irreducible. Otherwise, $V_{i}=V_{i}^{\prime} \oplus V_{i}^{\prime \prime}$ and $V_{i}^{\prime}, V_{i}^{\prime \prime}$ are dual $T$-modules. If they are isomorphic, $V_{i}$ is again a homogeneous component, and if they are not, each of $V_{i}^{\prime}, V_{i}^{\prime \prime}$ is a homogeneous component of $V$.

Suppose now that $\left|T_{i}\right|=1$. This can only happen if $G$ is symplectic or orthogonal and $q=2$. Then $V_{i}$ is acted on by $T$ trivially, $V_{i}$ is a hyperbolic plane, and $V^{T}$ is the sum of the $V_{i}$ with $T_{i}=1$. This proves the assertions. In particular, the uniqueness statements follow from these observations.

2.2. Classification of maximal tori in finite reductive groups. Let $q$ be a power of the prime $p$, and let $\mathbf{K}$ denote an algebraic closure of $\mathbb{F}_{p}$. We start with a connected reductive algebraic group $\mathbf{G}$ over $\mathbf{K}$, defined over $\mathbb{F}_{q}$, and denote by $F$ the corresponding Frobenius morphism. Closed, connected, F-stable subgroups of $\mathbf{G}$ will be denoted by boldface letters, and if $\mathbf{H}$ is such a subgroup, we write $H:=\mathbf{H}^{F}:=\{h \in \mathbf{H} \mid F(h)=h\}$ for the finite group of $F$-fixed points of $\mathbf{H}$. The pair $(\mathbf{G}, F)$, or simply the group $G=\mathbf{G}^{F}$, is called a finite reductive group or a finite group of Lie type.

To describe the maximal tori of $G$ up to $G$-conjugacy, we follow [6, Section 3.3]. Thus we fix an $F$-stable maximal torus $\mathbf{T}_{0}$ of $\mathbf{G}$, and let $W:=N_{\mathbf{G}}\left(\mathbf{T}_{0}\right) / \mathbf{T}_{0}$ denote the corresponding Weyl group of $\mathbf{G}$. (Notice that the results of [6. Section 3.3] are formulated for a maximally split torus $\mathbf{T}_{0}$, but that this assumption is not needed; see [7, 3.23].)

For every $w \in W$ we denote by $\dot{w}$ an element of $N:=N_{\mathbf{G}}\left(\mathbf{T}_{0}\right)$ mapping to $w$ under the natural epimorphism. For $t \in \mathbf{T}_{0}$ and $w \in W$ we let

$$
w_{t}:=\dot{w} t \dot{w}^{-1} \text {. }
$$


Clearly, the element ${ }^{w_{t}}$ does not depend on the particular choice of $\dot{w}$.

The $G^{F}$-classes of maximal tori in $G$ are in bijection with the $F$-conjugacy classes of $W$. These are the orbits on $W$ under the $F$-twisted $W$-action, also called $F$ conjugation, $w \mapsto v w F(v)^{-1}, v, w \in W$.

This bijection arises as follows. Let $w \in W$. By the Lang-Steinberg theorem, there is $g \in \mathbf{G}$ with $g^{-1} F(g)=\dot{w}$. Then $\mathbf{T}:={ }^{g} \mathbf{T}_{0}$ is $F$-stable and $T=\mathbf{T}^{F}=$ $g\left(\mathbf{T}_{0}^{w F}\right)$, where

$$
\mathbf{T}_{0}^{w F}:=\left\{\left.t \in \mathbf{T}_{0}\right|^{w} F(t)=t\right\} .
$$

Let $h \in \mathbf{G}$ with $h^{-1} F(h) \in N$. Then ${ }^{h} \mathbf{T}_{0}$ is $F$-stable and ${ }^{h} \mathbf{T}_{0}$ is conjugate to ${ }^{g} \mathbf{T}_{0}$ in $G$ if and only if the image of $h^{-1} F(h)$ in $W$ is $F$-conjugate to $w$ in $W$. We write $\mathbf{T}_{w}$ for any $F$-stable maximal torus of $\mathbf{G}$ which corresponds to the $F$-conjugacy class of $w \in W$ in the way described above, and we say that $\mathbf{T}_{w}$ arises from $\mathbf{T}_{0}$ by twisting with $w$.

Let $\mathbf{T}$ be an $F$-stable maximal torus of $\mathbf{G}$. We put $W(\mathbf{T}):=W_{\mathbf{G}}(\mathbf{T}):=$ $N_{\mathbf{G}}(\mathbf{T}) / \mathbf{T}$ (so that $W=W\left(\mathbf{T}_{0}\right)$ ). Then $F$ acts on $W(\mathbf{T})$, and we have $W(\mathbf{T})^{F} \cong$ $N_{\mathbf{G}}(\mathbf{T})^{F} / \mathbf{T}^{F}$ for the set of $F$-fixed points on $W(\mathbf{T})$ (see [6, Section 1.17]). If $\mathbf{T}=\mathbf{T}_{w}$ for some $w \in W$, then $W(\mathbf{T})^{F} \cong C_{W, F}(w)$, the $F$-centralizer of $w$ (see [6. Proposition 3.3.6]).

Let us write $\mathcal{S}(\mathbf{G})$ for the set of pairs $(\mathbf{T}, s)$, where $\mathbf{T}$ runs through the $F$ stable maximal tori of $\mathbf{G}$ and $s \in T$. We are interested in classifying $\mathcal{S}(\mathbf{G})$ up to $G$-conjugacy. For this purpose let

$$
\mathcal{P}:=\left\{(w, t) \mid w \in W, t \in \mathbf{T}_{0}^{w F}\right\} .
$$

As indicated above, an element $(w, t) \in \mathcal{P}$ determines a $G$-conjugacy class of elements of $\mathcal{S}(\mathbf{G})$. The Weyl group $W$ acts on $\mathcal{P}$ by $v \cdot(w, t):=\left(v w F(v)^{-1},{ }^{v} t\right)$ for $v \in W,(w, t) \in \mathcal{P}$. Two elements of $\mathcal{P}$ are in the same $W$-orbit if and only if they determine the same $G$-conjugacy class in $\mathcal{S}(\mathbf{G})$.

We will now give the specific examples to be used later on.

2.3. The unitary groups. Let $\mathbf{V}$ denote a vector space over $\mathbf{K}$ of dimension $d$, and fix a basis $v_{1}, v_{2}, \ldots, v_{d}$ of $\mathbf{V}$. We then identify $\mathbf{G}:=\mathrm{GL}(\mathbf{V})$ with the matrix group $\mathrm{GL}(d, \mathbf{K})$. To obtain the finite unitary groups, we let $F: \mathbf{G} \rightarrow \mathbf{G}$ be the Frobenius morphism defined by $F\left(a_{i j}\right):=\left(\left(a_{i j}^{q}\right)^{-1}\right)^{t}$ for $\left(a_{i j}\right) \in \mathbf{G}$. Then $G=\mathbf{G}^{F}=U(d, q) \leq \mathrm{GL}\left(d, q^{2}\right)$ with respect to the Hermitian form $\sum_{i=1}^{d} x_{i} y_{i}^{q}$ on

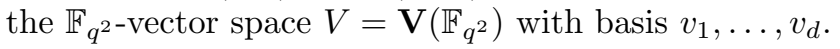

In this case we choose $\mathbf{T}_{0}$ to be the group of diagonal matrices of $\mathbf{G}$. (Thus $\mathbf{T}_{0}$ is not maximally split.) Then $N=N_{\mathbf{G}}\left(\mathbf{T}_{0}\right)$ is the group of monomial matrices and $W=N / \mathbf{T}_{0}$ can and will be identified with the subgroup of permutation matrices of $\mathbf{G}$. Thus $W$ is isomorphic to the symmetric group $S_{d}$ on $d$ letters, acting by permuting the basis vectors $v_{1}, \ldots, v_{d}$. Clearly, $F$ acts trivially on $W$.

The conjugacy classes of $W$ are parametrized by the partitions of $d$, via the cycle type of a permutation. Let $w \in W$. Assume that $w$ has $k$ cycles of even lengths $\mu_{1} \geq \cdots \geq \mu_{k}$, and $l$ cycles of odd lengths $\mu_{k+1} \geq \cdots \geq \mu_{k+l}$. We assume that $w=c_{1} c_{2} \cdots c_{k} c_{k+1} \cdots c_{k+l}$, where $c_{i}$ is a cycle of length $\mu_{i}$. For $1 \leq i \leq k+l$, let $\mathbf{V}_{i}$ denote the subspace of $\mathbf{V}$ spanned by the basis vectors moved by $c_{i}$ (or by the unique basis vector corresponding to $c_{i}$ if this is a 1-cycle), and put $\mathbf{G}_{i}:=\operatorname{GL}\left(\mathbf{V}_{i}\right)$. The subspace $\mathbf{V}_{i}$ has dimension $\mu_{i}$ and $\mathbf{V}=\mathbf{V}_{1} \oplus \cdots \oplus \mathbf{V}_{k+l}$. 
We embed $\mathbf{G}_{1} \times \cdots \times \mathbf{G}_{k+l}$ into $\mathbf{G}$ in the natural way. Note that each $\mathbf{G}_{i}$ is $F$ invariant, and that $G_{i}=\mathbf{G}_{i}^{F} \cong U\left(\mu_{i}, q\right)$, acting on $V_{i}=\mathbf{V}_{i}\left(\mathbb{F}_{q^{2}}\right)$, the $\mathbb{F}_{q^{2}}$-subspace of $\mathbf{V}_{i}$ generated by $\left\{v_{1}, \ldots, v_{d}\right\} \cap \mathbf{V}_{i}$.

Now choose $g_{i} \in \mathbf{G}_{i}$ with $g_{i}^{-1} F\left(g_{i}\right)=c_{i}, 1 \leq i \leq k+l$, and put $g:=g_{1} \times$ $\cdots \times g_{k+l}$. Then $g^{-1} F(g)=w$. Moreover, $\mathbf{T}:=\mathbf{T}_{w}:={ }^{g} \mathbf{T}_{0}=\mathbf{T}_{1} \times \cdots \times \mathbf{T}_{k+l}$, with $\mathbf{T}_{i}:={ }^{g} \mathbf{T}_{0, i}={ }^{g_{i}} \mathbf{T}_{0, i}$, where $\mathbf{T}_{0, i}:=\mathbf{T}_{0} \cap \mathbf{G}_{i}, 1 \leq i \leq k+l$. It follows that $T=\mathbf{T}^{F}=T_{1} \times \cdots \times T_{s+t}$, each $T_{i}$ acting on $V_{i}$.

Fix $i, 1 \leq i \leq k+l$, put $\mathbf{U}:=\mathbf{V}_{i}$ and $c:=c_{i}$. Let $u_{1}, \ldots, u_{m}$ be the basis vectors contained in $\mathbf{U}$, numbered in such a way that $c$ maps $u_{j}$ to $u_{j+1}, 1 \leq j \leq m$ (indices taken modulo $m$ ). Write $h\left(\zeta_{1}, \ldots, \zeta_{m}\right)$ for the element of $\mathbf{T}_{0, i}$ which acts on $u_{j}$ by multiplication with $\zeta_{j} \in \mathbf{K}^{\times}, 1 \leq j \leq m$. Then ${ }^{c} F\left(h\left(\zeta_{1}, \ldots, \zeta_{m}\right)\right)=$ $h\left(\zeta_{m}^{-q}, \zeta_{1}^{-q}, \ldots, \zeta_{m-1}^{-q}\right)$. Thus $h\left(\zeta_{1}, \ldots, \zeta_{m}\right)$ is fixed under the action of $c F$ if and only if $h\left(\zeta_{1}, \ldots, \zeta_{m}\right)=h\left(\zeta, \zeta^{-q}, \ldots, \zeta^{(-q)^{m-1}}\right)$ for some $\zeta \in \mathbf{K}$ with $\zeta^{(-q)^{m}}=\zeta$. It follows that $T_{i}$ is cyclic of order $q^{m}-1$, if $m$ is even, and of order $q^{m}+1$, if $m$ is odd. In the former case, $T_{i}$ fixes a maximal isotropic subspace of $V_{i}$, and in the latter case $T_{i}$ acts irreducibly on $V_{i}$.

We have thus constructed a $T$-decomposition of $V$ (see 2.1).

If $z \in C_{W, F}(w)$, then $z$ permutes the cycles $c_{i}$ of $w$. Hence $\dot{z}$ also permutes the tori $\mathbf{T}_{0, i}$, and so the corresponding element $g \dot{z} g^{-1} \in W(\mathbf{T})^{F}$ permutes the tori $\mathbf{T}_{i}$.

2.4. The symplectic and orthogonal groups. Let $\mathbf{V}$ be a vector space over $\mathbf{K}$ of dimension $d=2 n$ or $d=2 n+1$. We choose a basis

$$
v_{1}, v_{2}, \ldots, v_{n},\left[v_{0},\right] v_{n}^{\prime}, \ldots, v_{2}^{\prime}, v_{1}^{\prime}
$$

of $\mathbf{V}$ (where $v_{0}$ is not present if $d=2 n$ ). The typical element of $\mathbf{V}$ is denoted as $\left[x_{0} v_{0}\right]+\sum_{i=1}^{n} x_{i} v_{i}+x_{i}^{\prime} v_{i}^{\prime}$ with $x_{0}, x_{i}, x_{i}^{\prime} \in \mathbf{K}$ (and without the first summand if $d=2 n)$. Elements of $\mathrm{GL}(\mathbf{V})$ are written as matrices with respect to the basis (3).

If $d=2 n$, we define a symplectic form on $\mathbf{V}$ such that $v_{i}, v_{i}^{\prime}$ is a hyperbolic pair for all $1 \leq i \leq n$ and such that the planes $\left\langle v_{i}, v_{i}^{\prime}\right\rangle$ are pairwise orthogonal. Let $\mathbf{G}:=\operatorname{Sp}(\mathbf{V})$ denote the symplectic group with respect to this form. We usually identify the elements of $\mathbf{G}$ with their matrices with respect to the basis (3), so that $\mathbf{G}=\mathrm{Sp}(2 n, \mathbf{K}) \leq \mathrm{GL}(2 n, \mathbf{K})$. We let $F$ denote the standard Frobenius morphism of $\mathbf{G}$ mapping the matrix $\left(a_{i j}\right)$ to $\left(a_{i j}^{q}\right)$. Then $G=\mathbf{G}^{F}=\operatorname{Sp}(2 n, q) \leq \mathrm{GL}(2 n, q)$ with respect to the symplectic form $\sum_{i=1}^{n}\left(x_{i} y_{i}^{\prime}-x_{i}^{\prime} y_{i}\right)$ on the $\mathbb{F}_{q}$-vector space $V=\mathbf{V}\left(\mathbb{F}_{q}\right)$ with basis $v_{1}, \ldots, v_{n}, v_{n}^{\prime}, \ldots, v_{1}^{\prime}$.

If $d=2 n+1$, we define the orthogonal form $Q$ on $\mathbf{V}$ by $Q\left(x_{0} v_{0}+\sum_{i=1}^{n} x_{i} v_{i}+\right.$ $\left.x_{i}^{\prime} v_{i}^{\prime}\right):=x_{0}^{2}+\sum_{i=1}^{n} x_{i} x_{i}^{\prime}$. Let $\mathbf{G}:=\mathrm{SO}(\mathbf{V})=\mathrm{SO}(2 n+1, \mathbf{K}) \leq \mathrm{GL}(2 n+1, \mathbf{K})$ denote the special orthogonal group with respect to this form, and let $F$ be the standard Frobenius morphism of $\mathbf{G}$. Then $G=\mathbf{G}^{F}=\mathrm{SO}(2 n+1, q) \leq \mathrm{GL}(2 n+1, q)$ with respect to the orthogonal form $x_{0}^{2}+\sum_{i=1}^{n} x_{i} x_{i}^{\prime}$ on the $\mathbb{F}_{q}$-vector space $V=\mathbf{V}\left(\mathbb{F}_{q}\right)$ with basis $v_{1}, \ldots, v_{n}, v_{0}, v_{n}^{\prime}, \ldots, v_{1}^{\prime}$.

Now let $\mathbf{V}, \mathbf{G}, F$ be one of the two configurations introduced above. We choose $\mathbf{T}_{0}$ to be the group of diagonal matrices of $\mathbf{G}$. For $\zeta_{1}, \ldots, \zeta_{n} \in \mathbf{K}^{\times}$ we let $h\left(\zeta_{1}, \ldots, \zeta_{n}\right)$ denote the diagonal element of $\mathbf{G}$ which acts by multiplication with $\zeta_{i}$ on $v_{i}$, and by multiplication with $\zeta_{i}^{-1}$ on $v_{i}^{\prime}, 1 \leq i \leq n$. Thus $\mathbf{T}_{0}=\left\{h\left(\zeta_{1}, \ldots, \zeta_{n}\right) \mid \zeta_{1}, \ldots, \zeta_{n} \in \mathbf{K}^{\times}\right\}$. (If $\mathbf{G}$ is orthogonal, every element of $\mathbf{T}_{0}$ fixes $v_{0}$.) 
Let $W=N / \mathbf{T}_{0}$ with $N=N_{\mathbf{G}}\left(\mathbf{T}_{0}\right)$ denote the Weyl group of $\mathbf{G}$. Then $W$ is the Weyl group of type $C_{n}$, isomorphic to the wreath product of a cyclic group of order 2 with $S_{n}$. Clearly, $F$ acts trivially on $W$.

It is convenient to consider the faithful actions of $W$ on the character group $X:=X\left(\mathbf{T}_{0}\right):=\operatorname{Hom}\left(\mathbf{T}_{0}, \mathbf{K}^{\times}\right)$and on the cocharacter group $Y:=Y\left(\mathbf{T}_{0}\right):=$ $\operatorname{Hom}\left(\mathbf{K}^{\times}, \mathbf{T}_{0}\right)$ of $\mathbf{T}_{0}$. These are free abelian groups of rank $n$ with bases $\hat{e}_{1}, \ldots, \hat{e}_{n}$ defined by $\hat{e}_{i}\left(h\left(\zeta_{1}, \ldots, \zeta_{n}\right)\right)=\zeta_{i}$, and $e_{i}(\zeta)=h(1, \ldots, 1, \zeta, 1, \ldots, 1)$ (where $\zeta$ is on position $i$ ), respectively. The action of $W$ on $X$ and $Y$ fixes the sets $\left\{ \pm \hat{e}_{j} \mid 1 \leq j \leq\right.$ $n\}$ and $\left\{ \pm e_{j} \mid 1 \leq j \leq n\right\}$, respectively.

The set of conjugacy classes of $W$ is parametrized by the set of bipartitions of $n$. Let $w \in W$, viewed as a permutation group on $\left\{ \pm \hat{e}_{j} \mid 1 \leq j \leq n\right\}$. Then $w$ determines a bipartition of $n$ in the following way. There is a permutation $\pi=\pi(w)$ on $\{1, \ldots, n\}$ and a vector $\left(\sigma_{1}, \ldots, \sigma_{n}\right)$ of signs (i.e., $\sigma_{i} \in\{+1,-1\}$ for all $1 \leq i \leq n$ ) such that ${ }^{w} \hat{e}_{i}=\sigma_{i} \hat{e}_{\pi(i)}$ for all $1 \leq i \leq n$. The type of a cycle $\left(i_{1}, i_{2}, \ldots, i_{m}\right)$ of $\pi$ on $\{1, \ldots, n\}$ is the $\operatorname{sign} \sigma_{i_{1}} \sigma_{i_{2}} \cdots \sigma_{i_{m}}$. Let $\mu_{1} \geq \mu_{2} \geq \cdots \geq \mu_{k}$ denote the lengths of the cycles of type +1 of $\pi$, and $\nu_{1} \geq \nu_{2} \geq \cdots \geq \nu_{l}$ the lengths of the cycles of type -1 of $\pi$. Then the pair $(\mu, \nu)$ with $\mu:=\left(\mu_{1}, \ldots, \mu_{k}\right)$ and $\nu:=\left(\nu_{1}, \ldots, \nu_{l}\right)$ is a bipartition of $n$ which determines $w$ up to conjugacy in $W$. Clearly, every bipartition of $n$ arises in this way from a conjugacy class of $W$.

Let $w \in W$ correspond to the bipartition $(\mu, \nu)$ as above. Then $w=c_{1} c_{2} \ldots$ $c_{k} c_{k+1} \cdots c_{k+l}$ with pairwise commuting elements $c_{i} \in W$, such that $\pi\left(c_{i}\right)$ is a cycle of type +1 and length $\mu_{i}$ for $1 \leq i \leq k$, and a cycle of type -1 and length $\nu_{i-k}$ for $k+1 \leq i \leq k+l$. The set of elements of $\left\{ \pm \hat{e}_{j}\right\}$ moved by $c_{i}$ is invariant under multiplication by -1 , and these sets form a partition of $\left\{ \pm \hat{e}_{j} \mid 1 \leq j \leq n\right\}$. We obtain a decomposition

$$
X=X_{1} \oplus \cdots \oplus X_{k+l}
$$

into a direct sum of $w$-invariant, $w$-irreducible subgroups $X_{i}$ spanned by the orbits of $\langle w\rangle$ on $\left\{ \pm \hat{e}_{j}\right\}$. We have a corresponding decomposition

$$
Y=Y_{1} \oplus \cdots \oplus Y_{k+l} .
$$

For each $1 \leq i \leq k+l$, let $\mathbf{V}_{i}$ denote the subspace of $\mathbf{V}$ spanned by the basis vectors corresponding to the elements moved by $c_{i}$ (or to the two basis vectors $u, u^{\prime}$ corresponding to $c_{i}$ if this is a 1-cycle), and put $\mathbf{G}_{i}:=\operatorname{Sp}\left(\mathbf{V}_{i}\right)$ or $\mathbf{G}_{i}:=\operatorname{SO}\left(\mathbf{V}_{i}\right)$, respectively. The space $\mathbf{V}_{i}$ has dimension $2 \mu_{i}$ (with $\mu_{i}:=\nu_{i-k}$ for $i>k$ ), and $\mathbf{V}=\left[\mathbf{V}_{0} \oplus\right] \mathbf{V}_{1} \oplus \cdots \oplus \mathbf{V}_{k+l}$ (with $\mathbf{V}_{0}:=\left\langle v_{0}\right\rangle$ in the orthogonal case). We embed $\mathbf{G}_{1} \times \cdots \times \mathbf{G}_{k+l}$ into $\mathbf{G}$ in the natural way. Note that each $\mathbf{G}_{i}$ is $F$-invariant, and that $G_{i}=\mathbf{G}_{i}^{F} \cong \operatorname{Sp}\left(2 n_{i}, q\right)$ or $\mathrm{SO}^{ \pm}\left(2 n_{i}, q\right)$ (with $n_{i}=\mu_{i}$ or $\left.\nu_{i}\right)$, acting on $V_{i}=\mathbf{V}_{i}\left(\mathbb{F}_{q}\right)$, the $\mathbb{F}_{q}$-subspace of $\mathbf{V}_{i}$ generated by the basis vectors it contains.

Now choose $g_{i} \in \mathbf{G}_{i}$ with $g_{i}^{-1} F\left(g_{i}\right)=\dot{c}_{i}, 1 \leq i \leq k+l$, and put $g:=g_{1} \times \cdots \times$ $g_{k+l}$. If $1 \leq i \leq k$, the element $c_{i}$ lies in the stabilizer of the maximal isotropic subspace generated by $v_{1}, \ldots, v_{n}$ and we choose $g_{i} \in \mathbf{G}_{i}$ also fixing this space. Then $g^{-1} F(g)=w$. Moreover, $\mathbf{T}:={ }^{g} \mathbf{T}_{0}=\mathbf{T}_{1} \times \cdots \times \mathbf{T}_{k+l}$, with $\mathbf{T}_{i}:={ }^{g} \mathbf{T}_{0, i}={ }^{g_{i}} \mathbf{T}_{0, i}$, where $\mathbf{T}_{0, i}:=\mathbf{T}_{0} \cap \mathbf{G}_{i}, 1 \leq i \leq k+l$. It follows that $T=\mathbf{T}^{F}=T_{1} \times \cdots \times T_{k+l}$, each $T_{i}$ acting on $V_{i}$.

Fix $i, 1 \leq i \leq k+l$, put $\mathbf{U}:=\mathbf{V}_{i}$ and $c:=c_{i}$. Let $\hat{e}_{j_{1}}, \ldots, \hat{e}_{j_{m}}$ be the elements moved by $\pi(c)$, numbered in such a way that $\pi(c)$ maps $\hat{e}_{j_{r}}$ to $\hat{e}_{j_{r+1}} 1 \leq r \leq m$ (lower indices taken modulo $m$ ). For $1 \leq r \leq m$, put $u_{r}:=v_{j_{r}}$ and $u_{r}^{\prime}:=v_{j_{r}}^{\prime}$. Write $h\left(\zeta_{1}, \ldots, \zeta_{m}\right)$ for the element of $\mathbf{T}_{0, i}$ which acts on $u_{j}$ by multiplication 
with $\zeta_{j} \in \mathbf{K}^{\times}, 1 \leq j \leq m$. Suppose first that $i \leq k$. Then ${ }^{c} F\left(h\left(\zeta_{1}, \ldots, \zeta_{m}\right)\right)=$ $h\left(\zeta_{m}^{q}, \zeta_{1}^{q}, \ldots, \zeta_{m-1}^{q}\right)$. Thus $h\left(\zeta_{1}, \ldots, \zeta_{m}\right)$ is fixed under the action of $c F$ if and only if $h\left(\zeta_{1}, \ldots, \zeta_{m}\right)=h\left(\zeta, \zeta^{q}, \ldots, \zeta^{q^{m-1}}\right)$ for some $\zeta \in \mathbf{K}^{\times}$with $\zeta^{q^{m}}=\zeta$. It follows that $T_{i}$ is cyclic of order $q^{m}-1$. Moreover, $T_{i}$ fixes the maximal isotropic subspace spanned by $v_{1}, \ldots, v_{n}$, by our choice of $g_{i}$. Next assume that $k+1 \leq i \leq k+l$. By conjugating $c=c_{i}$ by a suitable element of $W$, we may and will assume that ${ }^{c} F\left(h\left(\zeta_{1}, \ldots, \zeta_{m}\right)\right)=h\left(\zeta_{m}^{-q}, \zeta_{1}^{q}, \ldots, \zeta_{m-1}^{q}\right)$. Thus the $c F$-fixed points on $\mathbf{T}_{0}$ are of the form $h\left(\zeta, \zeta^{q}, \ldots, \zeta^{q^{m-1}}\right)$ for some $\zeta \in \mathbf{K}^{\times}$with $\zeta^{q^{m}}=\zeta^{-1}$. Hence $T_{i}$ is cyclic of order $q^{m}+1$. Moreover, $T_{i}$ acts irreducibly on $V_{i}$.

Again, we have constructed a $T$-decomposition of $V$. As in the case of the unitary groups, we notice that the elements of $W(\mathbf{T})^{F}$ permute the tori $\mathbf{T}_{i}$.

2.5. Neutral maximal tori. We let $\mathbf{V}, \mathbf{G}, F$ be one of the configurations introduced in 2.3 or 2.4 and put $n=[(\operatorname{dim} \mathbf{V}) / 2]$ (the integer part). Thus $d=2 n$ or $2 n+1$ in the situation of Subsection 2.3 (and $n$ has the same meaning as in Subsection 2.4 if $\mathbf{G}$ is symplectic or orthogonal).

We call a maximal torus $T=\mathbf{T}^{F}$ of $G$ neutral, if no $V_{i}$ in the $T$-decomposition of $V$, as specified above, is an irreducible $T$-module.

Lemma 2.2. (1) If $(\mathbf{G}, F)$ is as in 2.3 and if $d=2 n+1$ is odd, then $G$ does not have any neutral maximal torus.

(2) Let $(\mathbf{G}, F)$ be an orthogonal group as in 2.4 , and let $\mathbf{T}$ be an $F$-stable maximal torus of $\mathbf{G}$. Consider the corresponding $T$-decomposition $V=V_{0} \oplus V_{1} \oplus \cdots \oplus V_{k+l}$ of $V$. If the Witt index of $V_{i}$ is less than $\left(\operatorname{dim} V_{i}\right) / 2$ for some $1 \leq i \leq k+l$, then $i>k$. In particular, $T$ is not neutral.

(3) If $(\mathbf{G}, F)$ is as in 2.3 or 2.4 , and if $d=2 n$ is even, then the $G$-conjugacy classes of neutral maximal tori of $G$ are in a bijective correspondence with the set of partitions of $n$.

Proof. We first prove (1) and (3). Suppose that we are in the situation of 2.3. Then the torus $\mathbf{T}_{w}$ is neutral if and only if $l=0$, i.e., if and only if all $\mu_{i}$ are even. In this case, $\left(\mu_{1} / 2, \ldots, \mu_{k} / 2\right)$ is a partition of $n=d / 2$.

A torus $\mathbf{T}_{w}$ in the situation of 2.4 is neutral if and only if the partition $\nu$ is empty. Hence such tori are in bijection with the set of bipartitions of $n$ of the form $(\mu,-)$, where $\mu$ runs through the partitions of $n$.

To prove (2), observe that for $i \leq k$, an irreducible $T$-submodule of $V_{i}$ is maximal singular of dimension $\left(\operatorname{dim} V_{i}\right) / 2$.

Lemma 2.3. Let $(\mathbf{G}, F)$ be as in 2.3 or 2.4 and let $\mathbf{T}$ be a neutral maximal torus in $\mathbf{G}$ corresponding to the partition $\left(1^{m_{1}}, 2^{m_{2}}, \ldots, n^{m_{n}}\right)$ of $n$. Then $\left|W(\mathbf{T})^{F}\right|=$ $\prod_{i=1}^{n}(2 i)^{m_{i}} m_{i} !$.

Proof. This follows directly from $W\left(\mathbf{T}_{w}\right)^{F} \cong C_{W, F}(w)$ and the well-known descriptions of the $F$-centralizers in the respective Weyl groups.

2.6. Some notation. We end this section by introducing some character theoretic notation, where the word character refers to a complex character of a finite group. Let $X$ and $Y$ be finite groups. We denote by $\rho_{X}$ and $1_{X}$ the regular and the trivial character of $X$. If $X$ has a unique cyclic quotient group of even order, we denote by $1_{X}^{-}$the non-trivial linear character of $X$ with values \pm 1 . For uniformity of some expressions, if $X$ is of odd order, we interpret $1_{X}^{-}$as $1_{X}$. If $\chi$ and $\psi$ are 
characters of $X$ and $Y$, respectively, $\chi \otimes \psi$ denotes their outer product, a character of $X \times Y$. In contrast, we use the symbol $\otimes$ to denote the (inner) tensor product of representations of $X$. If $Y$ is a subgroup of $X$, then $\chi_{Y}$ is the restriction of $\chi$ to $Y$, and $\psi^{X}$ the character of $X$ induced from $\psi$. Finally, the usual inner product of two complex class functions $\chi$ and $\psi$ of $X$ is denoted by $(\chi, \psi)$.

\section{Duality And GeOmetric CONJugacy}

Let $(\mathbf{G}, F)$ be a finite reductive group. We have to investigate the dual reductive group $\left(\mathbf{G}^{*}, F^{*}\right)$ to some extent. In particular, we wish to describe the pairs $(\mathbf{T}, \theta)$, where $\mathbf{T}$ is a maximal $F$-stable torus of $\mathbf{G}$, and $\theta$ is an irreducible (complex) character of $T$, up to conjugation in $G$. This is most conveniently done by passing to the dual group. We fix a maximal $F$-stable torus $\mathbf{T}_{0}$ of $\mathbf{G}$, and a maximal $F^{*}$-stable torus $\mathbf{T}_{0}^{*}$ of $\mathbf{G}^{*}$ satisfying the conditions of [6, Proposition 4.3.1]. In other words, $(\mathbf{G}, F)$ and $\left(\mathbf{G}^{*}, F^{*}\right)$ are in duality with respect to the pair $\left(\mathbf{T}_{0}, \mathbf{T}_{0}^{*}\right)$. Again, the assumption of [6], that the tori be maximally split, is not needed. In the following, we mark the objects associated with $\mathbf{G}^{*}$ with an asterisk.

3.1. Geometric conjugacy. We identify $X:=\operatorname{Hom}\left(\mathbf{T}_{0}, \mathbf{K}^{\times}\right)$with $Y^{*}:=$ $\operatorname{Hom}\left(\mathbf{K}^{\times}, \mathbf{T}_{0}^{*}\right)$ and $Y:=\operatorname{Hom}\left(\mathbf{K}^{\times}, \mathbf{T}_{0}\right)$ with $X^{*}:=\operatorname{Hom}\left(\mathbf{T}_{0}^{*}, \mathbf{K}^{\times}\right)$. Denote by $W:=W\left(\mathbf{T}_{0}\right)$ and $W^{*}:=W\left(\mathbf{T}_{0}^{*}\right)$ the Weyl groups of $\mathbf{G}$ and of $\mathbf{G}^{*}$, respectively.

The identification of $Y$ with $X^{*}$ yields an $F-F^{*}$-equivariant isomorphism

$$
\delta: \operatorname{Hom}\left(Y, \mathbf{K}^{\times}\right)=\operatorname{Hom}\left(X^{*}, \mathbf{K}^{\times}\right) \rightarrow \mathbf{T}_{0}^{*}
$$

of abelian groups. For the isomorphism $\operatorname{Hom}\left(X^{*}, \mathbf{K}^{\times}\right) \rightarrow \mathbf{T}_{0}^{*}$ see 6 , Propostion 3.1.2(i)]. As in [6, Proposition 4.2.3], there is an anti-isomorphism $W \rightarrow$ $W^{*}, w \mapsto w^{*}$, such that $\delta\left(w^{-1} \psi\right)=w^{*} \delta(\psi)$ for all $\psi \in \operatorname{Hom}\left(Y, \mathbf{K}^{\times}\right)$and $w \in W$.

Put

$$
\mathcal{Q}:=\left\{(w, \psi) \mid w \in W, \psi \in \operatorname{Hom}\left(Y, \mathbf{K}^{\times}\right), F\left({ }^{w^{-1}} \psi\right)=\psi\right\} .
$$

Then $W$ acts on $\mathcal{Q}$ by $v \cdot(w, \psi):=\left(v w F(v)^{-1},{ }^{v} \psi\right)$ for $v \in W,(w, \psi) \in \mathcal{Q}$, and there is a bijection

$$
\mathcal{Q} \rightarrow \mathcal{P}^{*}, \quad(w, \psi) \mapsto\left(F^{*}\left(w^{*}\right), \delta(\psi)\right) .
$$

(For the definition of $\mathcal{P}^{*}$ see (2).) One easily checks that $v \cdot(w, \psi)$ is mapped to $v^{*-1} .\left(F^{*}\left(w^{*}\right), \delta(\psi)\right)$. In particular, this map induces a bijection of the $W$-orbits in $\mathcal{Q}$ with the $W^{*}$-orbits in $\mathcal{P}^{*}$.

Let us write $\mathcal{T}(\mathbf{G})$ for the set of pairs $(\mathbf{T}, \theta)$, where $\mathbf{T}$ runs through the $F$-stable maximal tori of $\mathbf{G}$ and $\theta \in \operatorname{Irr}(T)$.

An element of $\mathcal{Q}$ gives rise to a $G$-conjugacy class of elements of $\mathcal{T}(\mathbf{G})$ as follows. Choose an isomorphism

$$
\Omega_{p^{\prime}} \rightarrow \mathbf{K}^{\times}
$$

where $\Omega_{p^{\prime}} \subseteq \mathbb{C}$ denotes the set of roots of unity of $p^{\prime}$-order (see [6, Proposition 3.1.3]). Let $(w, \psi) \in \mathcal{Q}$. The condition $F\left(w^{-1} \psi\right)=\psi$ is equivalent to $(w F-$ id) $Y \leq \operatorname{ker}(\psi)$. Hence $\psi$ may be viewed as an element of $\operatorname{Hom}\left(Y /(w F-\mathrm{id}) Y, \mathbf{K}^{\times}\right) \cong$ $\operatorname{Hom}\left(Y /(w F-\mathrm{id}) Y, \Omega_{p^{\prime}}\right)$. Moreover, $Y /(w F-\mathrm{id}) Y \cong \mathbf{T}_{0}^{w F}$ (see [6, Proposition 3.2.2]). We thus obtain a pair $(\mathbf{T}, \theta) \in \mathcal{T}(\mathbf{G})$ with $\mathbf{T}=\mathbf{T}_{w}$ and where $\psi$ is related to $\theta$ via an isomorphism

$$
\operatorname{Hom}\left(Y /(w F-\mathrm{id}) Y, \mathbf{K}^{\times}\right) \rightarrow \operatorname{Hom}\left(\mathbf{T}_{0}^{w F}, \Omega_{p^{\prime}}\right)=\operatorname{Hom}\left(\mathbf{T}_{0}^{w F}, \mathbb{C}^{\times}\right) .
$$


This construction yields a one-to-one correspondence between the set of $W$-orbits on $\mathcal{Q}$ and the set $G \backslash \mathcal{T}(\mathbf{G})$ of $G$-conjugacy classes on $\mathcal{T}(\mathbf{G})$. Through the bijection (5) and the considerations in 2.2. we obtain a one-to-one correspondence

$$
G \backslash \mathcal{T}(\mathbf{G}) \rightarrow G^{*} \backslash \mathcal{S}\left(\mathbf{G}^{*}\right),
$$

where $G^{*} \backslash \mathcal{S}\left(\mathbf{G}^{*}\right)$ denotes the set of $G^{*}$-conjugacy classes on $\mathcal{S}\left(\mathbf{G}^{*}\right)$. We say that $(\mathbf{T}, \theta) \in \mathcal{T}(\mathbf{G})$ and $\left(\mathbf{T}^{*}, s^{*}\right) \in \mathcal{S}\left(\mathbf{G}^{*}\right)$ are dual, if their respective conjugacy classes correspond via (7).

Finally, the bijection (5) yields an isomorphism

$$
\operatorname{Irr}\left(\mathbf{T}_{w}^{F}\right) \rightarrow \mathbf{T}_{F^{*}\left(w^{*}\right)}^{F^{*}}
$$

for every $w \in W$.

For $(\mathbf{T}, \theta) \in \mathcal{T}(\mathbf{G})$ we put $W(\mathbf{T})_{\theta}^{F}:=\left\{w \in W(\mathbf{T})^{F} \mid{ }^{w} \theta=\theta\right\}$ (for the definition of $W(\mathbf{T})$ see Subsection 2.2). Similarly, if $\left(\mathbf{T}^{*}, s^{*}\right) \in \mathcal{S}\left(\mathbf{G}^{*}\right)$, we put $W\left(\mathbf{T}^{*}\right)_{s^{*}}^{F^{*}}:=$ $\left\{w \in W\left(\mathbf{T}^{*}\right)^{F^{*}} \mid w^{*}=s^{*}\right\}$.

We will need the following lemma later on.

Lemma 3.1. Let $(\mathbf{G}, F)$ be a unitary group as in Subsection 2.3 or a symplectic group as in Subsection 2.4, and let $(\mathbf{T}, \theta) \in \mathcal{T}(\mathbf{G})$. Consider a T-decomposition of $V$ as constructed in these subsections.

Put $I:=\left\{1 \leq i \leq k+l \mid \theta_{i}=1_{T_{i}}^{-}\right\}$, and $J:=\left\{1 \leq i \leq k+l \mid \theta_{i} \neq 1_{T_{i}}^{-}\right\}$. Next, let $\mathbf{V}_{I}:=\bigoplus_{i \in I} \mathbf{V}_{i}$, and $\mathbf{V}_{J}:=\bigoplus_{i \in J} \mathbf{V}_{i}$, so that $\mathbf{V}=\mathbf{V}_{I} \oplus \mathbf{V}_{J}$.

Then the stabilizer in $\mathbf{G}$ of this decomposition equals $\mathbf{G}_{I} \times \mathbf{G}_{J}$, where $\mathbf{G}_{I}$ and $\mathbf{G}_{J}$ act as the identity on $\mathbf{V}_{J}$ and $\mathbf{V}_{I}$, respectively. Moreover, $\mathbf{T}=\mathbf{T}_{I} \times \mathbf{T}_{J}$ with the F-stable tori $\mathbf{T}_{I}:=\mathbf{T} \cap \mathbf{G}_{I}$ and $\mathbf{T}_{J}:=\mathbf{T} \cap \mathbf{G}_{J}$.

Put $\theta_{I}:=\theta_{T_{I}}$ and $\theta_{J}:=\theta_{T_{J}}$. Then

$$
W(\mathbf{T})_{\theta}^{F}=W_{\mathbf{G}_{I}}\left(\mathbf{T}_{I}\right)_{\theta_{I}}^{F} \times W_{\mathbf{G}_{J}}\left(\mathbf{T}_{J}\right)_{\theta_{J}}^{F} .
$$

Proof. First note that the stabilizer of the orthogonal decomposition $\mathbf{V}=\mathbf{V}_{I} \oplus \mathbf{V}_{J}$ equals $\mathbf{G}_{I} \times \mathbf{G}_{J}$, since $\mathbf{G}$ is a general linear or a symplectic group. Let $w \in W(\mathbf{T})_{\theta}^{F}$, and choose an inverse image $\dot{w} \in N_{G}(\mathbf{T})$ of $w$. Since $\dot{w}$ fixes $\theta$, and since $\dot{w}$ permutes the factors $T_{i}$ of $T$ by the final remarks of Subsections 2.3 and 2.4, it follows that $\dot{w}$ normalizes $T_{I}$ and $T_{J}$.

Now $\left|T_{j}\right|>1$ for each $j \in J$ and if $T_{j}=\left\langle t_{j}\right\rangle$, then $t_{j}$ does not have eigenvalue 1 on $V_{j}$. This implies that $V_{I}:=\sum_{i \in I} V_{i}$ equals the fixed space of $T_{J}$.

Since $\dot{w}$ normalizes $T_{J}$, it follows that $\dot{w}$ fixes $V_{I}$ and thus also $V_{J}=V_{I}^{\perp}$, and in turn it fixes $\mathbf{V}_{I}$ and $\mathbf{V}_{J}$. Thus $\dot{w}$ is contained in $\mathbf{G}_{I} \times \mathbf{G}_{J}$.

Hence $\dot{w} \in\left(\mathbf{G}_{I} \times \mathbf{G}_{J}\right)^{F}=\mathbf{G}_{I}^{F} \times \mathbf{G}_{J}^{F}$, and so $\dot{w}=\dot{w}_{I} \cdot \dot{w}_{J}$ with $\dot{w}_{I} \in N_{\mathbf{G}_{I}}\left(\mathbf{T}_{I}\right)^{F}$ and $\dot{w}_{J} \in N_{\mathbf{G}_{J}}\left(\mathbf{T}_{J}\right)^{F}$. Writing $w_{I}$ and $w_{J}$ for the images of $\dot{w}_{I}$ and $\dot{w}_{J}$ in $W_{\mathbf{G}}(\mathbf{T})^{F}$, respectively, we obtain $w_{I} \in W_{\mathbf{G}_{I}}\left(\mathbf{T}_{I}\right)_{\theta_{I}}^{F}$ and $w_{J} \in W_{\mathbf{G}_{J}}\left(\mathbf{T}_{J}\right)_{\theta_{J}}^{F}$, and hence the result.

3.2. Duality and $T$-decompositions. Let $(\mathbf{G}, F)$ be a unitary group as in 2.3 . or a symplectic group as in 2.4 If $(\mathbf{G}, F)$ is the finite unitary group as in 2.3, we may and will identify $(\mathbf{G}, F)$ with its dual $\left(\mathbf{G}^{*}, F^{*}\right)$ and put $\mathbf{T}_{0}=\mathbf{T}_{0}^{*}$. If $(\mathbf{G}, F)$ is the symplectic group as in 2.4 then $\left(\mathbf{G}^{*}, F^{*}\right)$ is the special orthogonal group of dimension $2 n+1$, also described in 2.4. As our reference torus $\mathbf{T}_{0}^{*}$ in $\mathbf{G}^{*}$ we take the torus denoted by $\mathbf{T}_{0}$ in 2.4 
Lemma 3.2. Let $(\mathbf{G}, F)$ be a unitary group as in 2.3 or a symplectic group as in 2.4. Suppose that $(\mathbf{T}, \theta) \in \mathcal{T}(\mathbf{G})$ and $\left(\mathbf{T}^{*}, s^{*}\right) \in \mathcal{S}\left(\mathbf{G}^{*}\right)$ are dual pairs. Then the following statements hold:

(a) $W(\mathbf{T})_{\theta}^{F} \cong W\left(\mathbf{T}^{*}\right)_{s^{*}}^{F^{*}}$.

(b) Let

$$
V=V_{1} \oplus \cdots \oplus V_{k} \oplus V_{k+1} \oplus \cdots \oplus V_{k+l}
$$

be a $T$-decomposition of $V$ as constructed in 2.3 or 2.4. Then there is a corresponding $T^{*}$-decomposition

$$
V^{*}=V_{0}^{*} \oplus V_{1}^{*} \oplus \cdots \oplus V_{k}^{*} \oplus V_{k+1}^{*} \oplus \cdots \oplus V_{k+l}^{*},
$$

of $V^{*}$ with $\operatorname{dim} V_{i}=\operatorname{dim} V_{i}^{*}$ for $1 \leq i \leq k+l$.

Consider the induced direct decompositions

$$
\left(\mathbf{T}_{1} \times \cdots \times \mathbf{T}_{k+l}, \theta_{1} \otimes \cdots \otimes \theta_{k+l}\right)
$$

of $(\mathbf{T}, \theta)$ and

$$
\left(\mathbf{T}^{*}, s^{*}\right)=\left(\mathbf{T}_{1}^{*} \times \cdots \times \mathbf{T}_{k+l}^{*}, s_{1}^{*} \times \cdots \times s_{k+l}^{*}\right)
$$

of $\left(\mathbf{T}^{*}, s^{*}\right)$. Then the order of $\theta_{i} \in \operatorname{Irr}\left(T_{i}\right)$ equals the order of $s_{i}^{*}$ as automorphism on $V_{i}^{*}$, for $1 \leq i \leq k+l$. In particular, $\theta_{i}=1_{T_{i}}^{-}$if and only if $s_{i}^{*}$ acts as -1 on $V_{i}^{*}$. Similarly, $\theta_{i}=1_{T_{i}}$ if and only if $s_{i}^{*}$ acts as the identity on $V_{i}^{*}$.

Proof. The isomorphism in (a) is derived in [6, p. 289].

By conjugating in $G$ and $G^{*}$, respectively, we may assume that $(\mathbf{T}, \theta)$ is constructed from $(w, \psi) \in \mathcal{Q}$ as in Subsection 3.1 and that $\left(\mathbf{T}^{*}, s^{*}\right)$ corresponds to $\left(w^{*}, \delta(\psi)\right)$ as in Subsection 2.2. (We remark that (a) now also follows from the fact that $W(\mathbf{T})_{\theta}^{F}$ and $W\left(\mathbf{T}^{*}\right)_{s^{*}}^{F^{*}}$ are isomorphic to the stabilizers of the pairs $(w, \psi) \in \mathcal{Q}$ and $\left(w^{*}, \delta(\psi)\right)$, respectively.) Notice that the conjugacy classes of $w$ and of $w^{*}$ are labelled by the same partition, respectively, bipartition (since inverse elements are conjugate). We construct $\mathbf{T}=\mathbf{T}_{w}, \mathbf{T}^{*}=\mathbf{T}_{w^{*}}^{*}$ and the corresponding decompositions of $V$ and $V^{*}$ as in 2.3 and 2.4 respectively. Considering the decompositions (4) of $Y$ arising from $w$, and of $\mathbf{T}_{0}^{*}$ arising from $w^{*}$, we obtain the following commutative diagram of abelian groups:

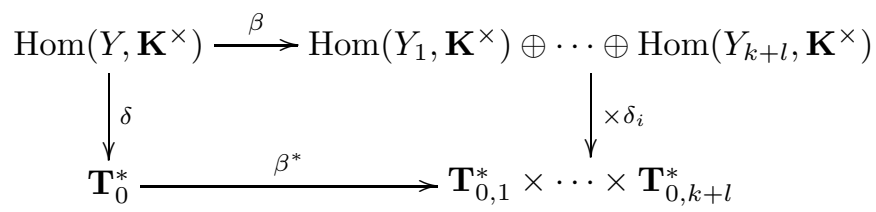

All isomorphisms are compatible with the actions of $\langle w, F\rangle$ in the top row and $\left\langle w^{*}, F^{*}\right\rangle$ in the bottom row. Writing $\beta(\psi)=\sum_{i=1}^{k+l} \psi_{i}$ with $\psi_{i} \in \operatorname{Hom}\left(Y_{i}, \mathbf{K}^{\times}\right)$, the characters $\theta_{i}$ correspond to $\psi_{i}$ and the elements $s_{i}^{*}$ correspond to $\delta_{i}\left(\psi_{i}\right)$ under the group isomorphisms (6). This gives the first result.

Finally, as $V_{i}^{*}$ has no proper non-degenerate $T_{i}^{*}$-invariant subspace, $s_{i}^{*}$ has order 2 if and only if it acts as -1 on $V_{i}^{*}$. Since $T_{i}$ is cyclic, the element $\theta_{i} \in \operatorname{Irr}\left(T_{i}\right)$ has order 2 if and only if $\theta_{i}=1_{T_{i}}^{-}$. The last statement is trivial. This completes the proof. 


\section{The CHARACTERS OF THE WeIL REPRESENTATIONS}

4.1. The ordinary case. Let $G=\operatorname{Sp}(2 n, q)$ with $q$ odd, or $U(d, q)$, with $q$ arbitrary. Let $V$ denote the natural module for $G$ and let $T$ be a maximal torus of G.

The standard reference for Weil representations is Gérardin [11, who computed their characters. If $G=U(d, q)$, there is a unique Weil representation of $G$ (up to equivalence). If $G=\operatorname{Sp}(2 n, q)$, there are two Weil representations of $G$ (see [11, Theorem 2.4(d)]), but the character values of the two Weil representations on semisimple elements are the same (see [11, Corollary 4.8.1]).

Let $\hat{\omega}^{(G)}$ denote the character of a Weil representation of $G$. If $G$ is symplectic, we put $\omega:=\omega^{(G)}:=\hat{\omega}^{(G)}$, and if $G$ is unitary, we put $\omega:=\omega^{(G)}:=1_{G}^{-} \cdot \hat{\omega}^{(G)}$. (Thus in the latter case, $\omega$ is not the character of Gérardin's Weil representation if $q$ is odd.)

The most important feature of the Weil representation is the multiplicative nature of its character. Namely, if $V=U \oplus U^{\prime}$ where $U$ and $U^{\prime}$ are non-degenerate and mutually orthogonal, then the embedding $H:=G_{U} \times G_{U^{\prime}}$ into $G$ gives $\omega_{H}=$ $\omega^{\left(G_{U}\right)} \otimes \omega^{\left(G_{U^{\prime}}\right)}$ (see [11, Corollaries 2.5, 3.4]).

Let $T$ be a maximal torus of $G$. Corresponding to a $T$-decomposition of $V$ we have an induced decomposition $T=T_{1} \times \cdots \times T_{k+l}$ of $T$, and a subgroup $H=G_{1} \times \cdots \times G_{k+l}$ of $G$. The above implies that $\omega_{H}=\omega_{1} \otimes \cdots \otimes \omega_{k+l}$, with $\omega_{i}:=\omega^{\left(G_{i}\right)}, 1 \leq i \leq k+l$.

Lemma 4.1. (a) Suppose that $k=1, l=0$. Then $\omega_{T}=\rho_{T}+1_{T}^{-}$.

(b) Suppose that $k=0, l=1$. Then $\omega_{T}=\rho_{T}-1_{T}^{-}$.

(c) In general, we have

$$
\omega_{T}=\left(\rho_{T_{1}}+1_{T_{1}}^{-}\right) \otimes \cdots \otimes\left(\rho_{T_{k}}+1_{T_{k}}^{-}\right) \otimes\left(\rho_{T_{k+1}}-1_{T_{k+1}}^{-}\right) \otimes \cdots \otimes\left(\rho_{T_{k+l}}-1_{T_{k+l}}^{-}\right) .
$$

Proof. The statements in (a) and (b) can be derived from [11, Corollaries 4.8.1, 4.8.2]. The last statement follows from these.

4.2. The modular case. We change the point of view and consider instead the $p$-modular version of the Weil representation. If $p>2$, this is just the Brauer reduction modulo $p$ of the Weil representation. If $G=\operatorname{Sp}(2 n, q)$ and $p=2$, the Weil representation does not exist, but it has been shown by the second author in [19], that the analogue of its Brauer reduction modulo 2 does exist, and that this is exactly the generalized spinor representation of $G$. If $q=2$, this is the usual spinor representation.

Let $q$ be a power of 2 and let $\mathbf{K}$ denote an algebraic closure of the finite field $\mathbb{F}_{q}$. Let $\mathbf{G}=\operatorname{Sp}(2 n, \mathbf{K})$ be the symplectic group of degree $2 n$ over $\mathbf{K}$ as introduced in Subsection 2.4 and let $F$ be the standard Frobenius map of $\mathbf{G}$ raising every matrix entry of $\mathbf{G}$ to its $q$ th power.

To introduce the generalized spinor representation of $G=\operatorname{Sp}(2 n, q)$, we recall some notions of algebraic group theory. Let $\lambda_{1}, \ldots, \lambda_{n}$ be the fundamental weights of $\mathbf{G}$ (ordered as in Bourbaki [4). An integer linear combination $\sum a_{i} \lambda_{i}$ is called a weight of $\mathbf{G}$, and the weights with $a_{i} \geq 0$ for $i=1, \ldots, n$ are called dominant. There is a canonical bijective correspondence between the dominant weights and the equivalence classes of rational irreducible representations of $\mathbf{G}$, and for a dominant weight $\nu$ we denote by $\phi_{\nu}$ the irreducible representation of $\mathbf{G}$ corresponding to $\nu$. We set $\sigma_{n}=\left(\phi_{(q-1) \lambda_{n}}\right)_{G}$ and call $\sigma_{n}$ the generalized spinor representation of $G$, 
while the spinor representation is $\left(\phi_{\lambda_{n}}\right)_{G}$. To avoid confusion we sometimes use the notation $\sigma_{n, q}$ for $\sigma_{n}$.

4.2.1. The Weil representation of the extrasymplectic group. Despite the fact that the representation $\sigma_{n}$ is explicitly constructed, its Brauer character does not seem to have been computed. We need to do this and, moreover, to express it in terms of characters of the maximal tori in $G$. We could do this by straightforward computations but it is more conceptual to connect this with complex representations of extraspecial 2-groups.

So we start with extraspecial groups. For a natural number $n$ there are two extraspecial groups of order $2^{2 n+1}$ which we denote by $E_{n}^{+}$and $E_{n}^{-}$. The center $Z$ of each of them is of order 2. The central quotients are elementary abelian 2-groups. Let $C_{4}$ denote the cyclic group of order 4 and let $E_{n}$ be the central product $C_{4} \cdot E_{n}^{+}$ (with common subgroup of order 2). Then $C_{4} \cdot E_{n}^{+}=C_{4} \cdot E_{n}^{-}$, so $E_{n}$ contains $E_{n}^{+}$ and $E_{n}^{-}$as subgroups of index 2. We denote the central quotient by $V_{n}$ in all three cases. Then the mapping $x Z \mapsto x^{2}$ defines a non-degenerate quadratic form on $V_{n}$ and the two forms corresponding to $E_{n}^{+}$and $E_{n}^{-}$are non-equivalent. The mapping $x Z \times y Z \mapsto[x, y]$ for $x, y \in E_{n}$ defines a non-degenerate alternating form on $V_{n}$ which is the polarization of both quadratic forms. Details can be found in [8, page 80]. Furthermore, Aut $E_{n}^{+} / \operatorname{Inn} E_{n}^{+} \cong O^{+}(2 n, 2)$, Aut $E_{n}^{-} / \operatorname{Inn} E_{n}^{-} \cong O^{-}(2 n, 2)([8$, Theorem 20.8]) and Aut $E_{n} / \operatorname{Inn} E_{n} \cong \operatorname{Sp}(2 n, 2) \times C_{2}$. We denote by Aut $^{0} E_{n}$ the subgroup of Aut $E_{n}$ consisting of the automorphisms acting trivially on the center. So $\mathrm{Aut}^{0} E_{n} / \operatorname{Inn} E_{n} \cong \operatorname{Sp}(2 n, 2)$.

It is also well known that every faithful complex irreducible representation of $E_{n}$ has degree $2^{n}$, and its character $\chi$ vanishes on all non-central elements. As elements of the center of $E_{n}$ are represented by scalar matrices, there are exactly two non-equivalent faithful irreducible representations of $E_{n}$ which are dual to each other. We denote any one of them by $\eta$. Let $\alpha$ be an automorphism of $E_{n}$ acting trivially on the center. Then $\eta^{\alpha}=\eta$. It follows that $\eta(\alpha(x))=g \eta(x) g^{-1}$ for some $g \in \mathrm{GL}\left(2^{n}, \mathbb{C}\right)$. As $g$ is determined by $\alpha$ up to a scalar multiple, the mapping Aut $^{0} E_{n} \rightarrow \mathrm{GL}\left(2^{n}, \mathbb{C}\right)$ obtained from this provides a projective representation $\pi$ of Aut ${ }^{0} E_{n}$ into $\mathrm{GL}\left(2^{n}, \mathbb{C}\right)$. An irreducible projective representation of a finite group can be obtained from an ordinary representation of a central extension. It turns out that a central extension of $\mathrm{Aut}^{0} E_{n}$ by a cyclic group of order 4 is sufficient. Thus, there exists a group $R=R(n, 2)$ with normal subgroup $E_{n}$ such that $R / E_{n} \cong$ $\operatorname{Sp}(2 n, 2)$, and an irreducible representation $\eta$ of $R$ of degree $2^{n}$ such that $\eta_{E_{n}}$ is irreducible.

It is well known that the group $\operatorname{Sp}\left(2 m, 2^{k}\right)$ is isomorphic to a subgroup of $\mathrm{Sp}(2 m k, 2)$. We fix an embedding $\operatorname{Sp}\left(2 m, 2^{k}\right) \rightarrow \operatorname{Sp}(2 m k, 2)$ and denote by $\operatorname{ESp}(2 m, q)$ for $q=2^{k}$ the preimage of $\operatorname{Sp}(2 m, q)$ in $R=R(m k, 2)$. We call $\operatorname{ESp}(2 m, q)$ the extrasymplectic group and use the term "Weil character" for the character of its irreducible representation of degree $2^{n}=q^{m}$. The Weil character depends on $\eta$ which is immaterial for what follows as we are only interested in the values of $\eta$ at odd order elements. These are independent of the choice of $\eta$.

Remark 4.2. (1) Usually the Weil character is considered for symplectic groups in odd characteristic. However, there is a strong similarity between the odd characteristic Weil character at semisimple elements and the above introduced Weil character for the extrasymplectic group at semisimple elements. Observe that $\operatorname{ESp}(2 m, q)$ is 
not split over $E_{n}$ so one cannot restrict $\eta$ to $\operatorname{Sp}(2 m, q)$ in contrast to the case of odd $q$.

(2) The existence of the above projective representation of $\mathrm{Aut}^{0} E_{n}$ was probably shown first in Suprunenko [17, Theorem 11], but he deals with the linear group $\eta\left(E_{n}\right) \cdot S$ where $S$ is the group of all non-zero scalar matrices. The observation that the symplectic group appears already as $\mathrm{Aut}^{0} E_{n} / \operatorname{Inn} E_{n}$ was probably first done by Isaacs [14, Section 4]. Isaacs also computes the character of $\eta$ at odd order elements, but we need to transform the information to a more convenient shape.

The following useful fact demonstrates the multiplicative nature of the Weil representations.

Lemma 4.3. Let $\eta_{m}$ be a Weil representation of the extrasymplectic group $\operatorname{ESp}(2 m, q)$ and let $H$ be an odd order subgroup.

Let $\lambda: \operatorname{ESp}(2 m, q) \rightarrow \operatorname{Sp}(2 m, q)$ be the natural projection and let $V$ be the natural module for $\operatorname{Sp}(2 m, q)$. Let $h \in \operatorname{ESp}(2 m, q)$ be of odd order. Suppose that $\lambda(h)$ preserves an orthogonal decomposition $V=V_{1} \oplus V_{2}$ and let $m_{i}=\operatorname{dim} V_{i}$ for $i=1,2$. Then $h=h_{1} h_{2}$ where $h_{1}, h_{2} \in \operatorname{ESp}(2 m, q), h_{1} h_{2}=h_{2} h_{1}$ and $\lambda\left(h_{1}\right)$ (respectively, $\lambda\left(h_{2}\right)$ ) acts trivially on $V_{2}$ (respectively, on $\left.V_{1}\right)$, and $\eta_{m}(h)=\eta_{m_{1}}\left(h_{1}\right) \cdot \eta_{m_{2}}\left(h_{2}\right)$.

Proof. This is contained in [14, Lemma 5.5].

Lemma 4.4. Suppose that $n>1$, let $\eta$ be an irreducible representation of $R=$ $\operatorname{ESp}(2 n, 2)$ as described above, and let $T \subset \operatorname{Sp}(2 n, 2)$ be a maximal cyclic torus of order $2^{n}+\varepsilon$ where $\varepsilon=1$ or -1 . Let $T^{\prime}$ be any subgroup of $R$ such that $\left|T^{\prime}\right|=|T|$ and $T^{\prime} E_{n} / E_{n}=T$. Then $\chi_{T^{\prime}}=\rho_{T^{\prime}}+\varepsilon \cdot 1_{T^{\prime}}$ where $\chi$ is the character of $\eta$, that is, the Weil character of $R$.

Proof. This is a particular case of [8, Theorem 9.18], however, we have to refine a few details. First, Theorem 9.18 in [8] is stated for an extraspecial group in place of $E_{n}$. However, it is known that $T$ is contained either in $O^{+}(2 n, 2)$ or in $O^{-}(2 n, 2)$ and we can use the result for extraspecial groups. Second, Theorem 9.18 in [8] claims that $\chi_{T^{\prime}}=\rho_{T^{\prime}}+\varepsilon \cdot \tau$ where $\tau$ is some linear character of $T^{\prime}$. To deduce that in our situation $\tau=1_{T^{\prime}}$, observe that $R$ is perfect (unless $n \leq 2$ ) and hence $\operatorname{det} \eta(t)=1$ for any $t \in T^{\prime}$. This is also true for $n=2$ as $\operatorname{Sp}(4,2)$ has a simple subgroup of index 2 , so $T^{\prime}$ belongs to the derived subgroup of $R$. As det $\eta(t)=\tau(t)$, the claim follows.

We fix an embedding $e: \operatorname{Sp}\left(2 m, 2^{k}\right) \rightarrow \operatorname{Sp}(2 n, 2)$ where $n=m k$ and denote by $\operatorname{ESp}(2 m, q)$ the preimage of $\operatorname{Sp}\left(2 m, 2^{k}\right)$ in $R=R(m k, 2)$. Moreover, if $T$ is a maximal torus in $\operatorname{Sp}\left(2 m, 2^{k}\right)$, then $e(T)$ is a maximal torus in $\operatorname{Sp}(2 n, 2)$, and $e\left(T_{1}\right) \times \cdots \times e\left(T_{k+l}\right)$ is an $e(T)$-decomposition of $e(T)$. Then Lemmas 4.4 and 4.3 yield the following result.

Proposition 4.5. Let $T$ be a maximal torus in $\operatorname{Sp}(2 n, 2)$, and let $T=T_{1} \times \cdots \times$ $T_{k} \times T_{k+1} \times \cdots \times T_{k+l}$ be a $T$-decomposition such that $\left|T_{i}\right|=2^{n_{i}}-1$ for $i \leq k$ and $\left|T_{i}\right|=2^{n_{i}}+1$ for $i>k$. Let $T^{\prime}, T_{i}^{\prime}$ be subgroups of $R$ such that $\left|T^{\prime}\right|=|T|,\left|T_{i}^{\prime}\right|=\left|T_{i}\right|$ for $1 \leq i \leq k+l$, and $T^{\prime} E_{n} / E_{n}=T, T_{i}^{\prime} E_{n} / E_{n}=T_{i}$. Let $\chi$ be the character of $\eta$. Then

$$
\chi_{T^{\prime}}=\left(\rho_{T_{1}^{\prime}}+1_{T_{1}^{\prime}}\right) \otimes \cdots \otimes\left(\rho_{T_{k}^{\prime}}+1_{T_{k}^{\prime}}\right) \otimes\left(\rho_{T_{k+1}^{\prime}}-1_{T_{k+1}^{\prime}}\right) \otimes \cdots \otimes\left(\rho_{T_{k+l}^{\prime}}-1_{T_{k+l}^{\prime}}\right) .
$$

Furthermore, this is true for maximal tori in $\operatorname{Sp}\left(2 m, 2^{k}\right) \subset \operatorname{Sp}(2 n, 2)$ where $n=m k$. 
Remark 4.6. It follows that $\chi_{T^{\prime}}$ is real valued and moreover, that $\chi(g)$ is a real number for every $g$ of odd order, as the projection of $g$ in $\operatorname{Sp}(2 n, 2)$ belongs to some maximal torus of $\operatorname{Sp}(2 n, 2)$.

4.2.2. The Brauer character of $\sigma_{n}$. Recall that $\lambda_{1}, \ldots, \lambda_{n}$ denote the fundamental weights of $\mathbf{G}$; for uniformity of some formulas below we set $\lambda_{0}=0$. We often use, without accurate reference, Steinberg's famous theorem saying that every irreducible representation of $G$ is of shape $\left(\phi_{\nu}\right)_{G}$ where $\nu$ is a $q$-restricted dominant weight, and conversely $\left(\phi_{\nu}\right)_{G}$ is irreducible for every $q$-restricted dominant weight $\nu$ of $\mathbf{G}$. Recall that a dominant weight $\nu=a_{1} \lambda_{1}+\cdots+a_{n} \lambda_{n}$ is called $q$-restricted if $0 \leq a_{i} \leq q-1$ (here $a_{1}, \ldots, a_{n}$ are integers). In addition, if $\nu$ is not 2-restricted, then $\phi_{\nu}$ can be expressed as the tensor product of 2-restricted irreducible representations twisted by the Frobenius morphism as follows. Let $q=2^{k}$ and let $a_{i}=\sum_{j=0}^{k-1} 2^{j} b_{i j}$ be the 2-adic expansion of $a_{i}$. Let $\nu_{j}=\sum_{i} b_{i j} \lambda_{i}$. Then $\phi_{\nu}=\phi_{\nu_{0}} \otimes F_{0}\left(\phi_{\nu_{1}}\right) \otimes \cdots \otimes F_{0}^{k-1}\left(\phi_{\nu_{k-1}}\right)$ where $F_{0}$ is the standard Frobenius morphism of $\mathbf{G}$ induced by the mapping $x \mapsto x^{2}$ for $x \in \mathbf{K}$ (so that $F=F_{0}^{k}$ ). In particular, $\phi_{(q-1) \lambda_{n}}=\phi_{\lambda_{n}} \otimes F_{0}\left(\phi_{\lambda_{n}}\right) \otimes \cdots \otimes F_{0}^{k-1}\left(\phi_{\lambda_{n}}\right)$; this fact will be also used without precise reference.

Lemma 4.7 ([19, Lemma 1.13]). Let e $: \operatorname{Sp}(2 n, \mathbf{K}) \rightarrow \operatorname{Sp}(2 n k, \mathbf{K})$ be the embedding defined by $g \mapsto \operatorname{diag}\left(g, F_{0}(g), \ldots, F_{0}^{k-1}(g)\right)$ for $g \in \operatorname{Sp}(2 n, \mathbf{K})$ (this is called a Frobenius embedding in [19]). Then the restriction of $\phi_{\lambda_{n k}}$ to $e(\operatorname{Sp}(2 n, \mathbf{K}))$ is irreducible and coincides with $\phi_{\lambda_{n}} \otimes F_{0}\left(\phi_{\lambda_{n}}\right) \otimes \cdots \otimes F_{0}^{k-1}\left(\phi_{\lambda_{n}}\right)=\phi_{(q-1) \lambda_{n}}$. Here, $\phi_{\lambda_{n k}}$ is the irreducible representation of $\operatorname{Sp}(2 n k, \mathbf{K})$ corresponding to the fundamental weight $\lambda_{n k}$, while $\phi_{\lambda_{n}}$ and $\phi_{(q-1) \lambda_{n}}$ refer to the group $\mathbf{G}=\operatorname{Sp}(2 n, \mathbf{K})$.

Corollary 4.8. The restriction $\left(\sigma_{n k, 2}\right)_{\operatorname{Sp}(2 n, q)}$ is equivalent to $\sigma_{n, q}$. (Here, $\sigma_{n k, 2}$ is the spinor representation of $\operatorname{Sp}(2 n k, 2)$ and $\operatorname{Sp}(2 n, q)$ is viewed as a subgroup of $\operatorname{Sp}(2 n k, 2)$ under an embedding obtained by regarding $\mathbb{F}_{q}$ as a vector space over $\left.\mathbb{F}_{2}.\right)$

Proof. Let $\mathbf{V}$ be the natural module for $\operatorname{Sp}(2 n k, \mathbf{K})$-module (that is, the one of highest weight $\left.\lambda_{1}\right)$. Then $\mathbf{V}_{\operatorname{Sp}(2 n, q)}$ is reducible; in fact, $\mathbf{V}_{\operatorname{Sp}(2 n, q)} \cong V_{n} \oplus F_{0}\left(V_{n}\right) \oplus$ $\cdots \oplus F_{0}^{k-1}\left(V_{n}\right)$ where $V_{n}$ is the natural $\operatorname{Sp}(2 n, q)$-module. So the result follows from Lemma 4.7 .

Proposition 4.9 ([19, Theorem 3.10]). The Brauer reduction modulo 2 of $\eta$ is irreducible and equivalent to the inflation of $\sigma_{n, 2}$ to $R=R(n, 2)$.

Corollary 4.10. The Brauer character of $\sigma_{n, 2}$ is real and coincides with the character of $\eta$ at elements of odd order.

Proposition 4.11. Let $q=2^{k}$. The Brauer reduction modulo 2 of $\eta$ is irreducible and equivalent to the inflation of $\sigma_{n, q}$ to $\operatorname{ESp}(2 n, q)$.

Proof. This is not explicitly stated in 19, but follows from Corollary 4.8. Indeed, by Proposition 4.9. the reduction of $\eta$ modulo 2 coincides with $\left(\sigma_{n k, 2}\right)_{\operatorname{Sp}(2 m, q)}$, which is $\sigma_{n, q}$ by Corollary 4.8 .

Proposition 4.12. Let $T$ be a maximal torus of $\operatorname{Sp}(2 n, q)$ and let $T=T_{1} \times \cdots \times$ $T_{k} \times T_{k+1} \times \cdots \times T_{k+l}$ be a $T$-decomposition such that $\left|T_{i}\right|=q^{n_{i}}-1$ for $i \leq k$ and $\left|T_{i}\right|=q^{n_{i}}+1$ for $i>k$. Let $\omega$ be the Brauer character of $\sigma_{n, q}$. Then

$\omega_{T}=\left(\rho_{T_{1}}+1_{T_{1}}\right) \otimes \cdots \otimes\left(\rho_{T_{k}}+1_{T_{k}}\right) \otimes\left(\rho_{T_{k+1}}-1_{T_{k+1}}\right) \otimes \cdots \otimes\left(\rho_{T_{k+l}}-1_{T_{k+l}}\right)$.

Proof. This follows from Propositions 4.5 and 4.11 
Corollary 4.13. Let $g \in \operatorname{Sp}(2 n, q)$ be an odd order element. Then $\omega(g)^{2}=q^{N(V ; g)}$ where $V$ is the natural $\operatorname{Sp}(2 n, q)$-module and $N(V ; g)$ the dimension of the 1 eigenspace of $g$ on $V$.

Proof. This can be deduced from Lemma 4.4 but is also available in Isaacs [14, Theorem 3.5].

4.3. Multiplicities in $\omega_{T}$. We return to the general situation. Namely, $G=$ $\operatorname{Sp}(2 n, q)$ or $U(d, q)$ with $q$ arbitrary. If $G=\operatorname{Sp}(2 n, q)$ and $q$ is even, we let $\omega$ denote the (Brauer) character of $G$ of the representation $\sigma_{n, q}$ as in Subsection 4.2 , Otherwise, $\omega$ denotes the character of a Weil representation of $G$ as introduced in Subsection 4.1. We let $T$ be a maximal torus of $G$ and consider a $T$-decomposition

$$
T=T_{1} \times \cdots \times T_{k} \times T_{k+1} \times \cdots \times T_{k+l}
$$

as in Subsection 2.1 .

Let $\theta \in \operatorname{Irr}(T)$. Then $\theta=\theta_{1} \otimes \cdots \otimes \theta_{k+l}$ with unique $\theta_{i} \in \operatorname{Irr}\left(T_{i}\right), 1 \leq i \leq k+l$. If $k=1$ and $l=0$, then we see from Lemma 4.1(a) and Proposition 4.12, respectively, that the multiplicity of every $\theta \in \operatorname{Irr}(T)$ in $\omega_{T}$ equals 1 , except for the character $1_{T}^{-}$, which has multiplicity 2. (Recall our convention that $1_{T}^{-}$stands for $1_{T}$ if $T$ has odd order, i.e., if $q$ is even.) Similarly, if $k=0$ and $l=1$, then Lemma 4.1(b), respectively, Proposition 4.12 implies that the multiplicity of every $\theta \in \operatorname{Irr}(T)$ in $\omega_{T}$ equals 1 , except for the character $1_{T}^{-}$, which has multiplicity 0 . In general, let $k(\theta)$ be the number of $i \leq k$ such that $\theta_{i}=1_{T_{i}}^{-}$. It follows from Lemma 4.1(c) that the multiplicity of $\theta \in \operatorname{Irr}(T)$ in $\omega_{T}$ equals $2^{k(\theta)}$, unless there is $j$ such that $\theta_{k+j}=1_{T_{k+j}}^{-}$, in which case $\theta$ does not occur in $\omega_{T}$. Thus we have proved the following.

Lemma 4.14. Let $\theta=\theta_{1} \otimes \cdots \otimes \theta_{k+l}$ be an irreducible character of $T=T_{1} \times \cdots \times$ $T_{k+l}$.

(1) If $\theta_{k+j}=1_{T_{k+j}}^{-}$for some $j>0$, then $\theta$ does not occur as an irreducible constituent of $\omega_{T}$ (that is, $\left(\omega_{T}, \theta\right)=0$ ).

(2) Suppose that $\theta_{k+j} \neq 1_{T_{k+j}}^{-}$for every $j=1, \ldots, l$. Let $k(\theta)$ be the number of $0 \leq i \leq k$ such that $\theta_{i}=1_{T_{i}}^{-}$. Then $\left(\omega_{T}, \theta\right)=2^{k(\theta)}$.

(3) Suppose that $\theta_{i} \neq 1_{T_{i}}^{-}$for every $1 \leq i \leq k+l$. Then $\left(\omega_{T}, \theta\right)=1$.

Note that the statements above remain true in case $G$ is a group of characteristic 2 , if $1_{T_{i}}^{-}$is replaced by $1_{T_{i}}$ throughout, in consistency with our convention.

\section{The Product $\omega \cdot$ St}

In this section we prove Theorem 1.2 for the symplectic and unitary groups.

Let $(\mathbf{G}, F)$ be a unitary group as in 2.3 or a symplectic group as in 2.4. We denote by $\left(\mathbf{G}^{*}, F^{*}\right)$ a reductive group dual to $(\mathbf{G}, F)$. Let $S^{*}$ denote the set of $G^{*}$-conjugacy classes of semisimple elements of $G^{*}$. We write $\left(s^{*}\right)$ for the element of $S^{*}$ containing $s^{*} \in G^{*}$. For each semisimple $s^{*} \in G^{*}$ we choose a set $\kappa\left(s^{*}\right)$ of representatives for the $G$-orbits in

$$
\left\{(\mathbf{T}, \theta) \in \mathcal{T}(\mathbf{G}) \mid(\mathbf{T}, \theta) \text { is dual to }\left(\mathbf{T}^{*}, s^{*}\right) \in \mathcal{S}\left(\mathbf{G}^{*}\right)\right\}
$$

(see (7)).

By St $=\mathrm{St}_{G}$ we denote the Steinberg character of $G=\mathbf{G}^{F}$ and by $\omega$ the class function introduced in Section 4. Then $\omega \cdot$ St is a character of $G$ vanishing on all 
$p$-singular elements. It is known that every such class function is uniform, that is, a linear combination of characters $R_{\mathbf{T}, \theta}$ (see [7, page 89]).

The argument in [6, p. 242] shows that

$$
\omega \cdot \mathrm{St}=\sum_{\left(s^{*}\right) \in S^{*}} \sum_{(\mathbf{T}, \theta) \in \kappa\left(s^{*}\right)} \frac{\varepsilon_{\mathbf{G}} \varepsilon_{\mathbf{T}}\left(\omega_{T}, \theta\right)}{\left|W(\mathbf{T})_{\theta}^{F}\right|} R_{\mathbf{T}, \theta} .
$$

For each $\left(s^{*}\right) \in S^{*}$ consider the partial sum

$$
\pi_{s^{*}}:=\sum_{(\mathbf{T}, \theta) \in \kappa\left(s^{*}\right)} \frac{\varepsilon_{\mathbf{G}} \varepsilon_{\mathbf{T}}\left(\omega_{T}, \theta\right)}{\left|W(\mathbf{T})_{\theta}^{F}\right|} R_{\mathbf{T}, \theta},
$$

as well as the class function

$$
\rho_{s^{*}}=\sum_{(\mathbf{T}, \theta) \in \kappa\left(s^{*}\right)} \frac{\varepsilon_{\mathbf{G}} \varepsilon_{\mathbf{T}}}{\left|W(\mathbf{T})_{\theta}^{F}\right|} R_{\mathbf{T}, \theta} .
$$

Lemma 5.1. Let $\chi_{\left(s^{*}\right)}$ be the class function introduced by Digne and Michel in [7, Definition 14.10]. Then $\chi_{\left(s^{*}\right)}=\rho_{s^{*}}$ if $C_{\mathbf{G}^{*}}\left(s^{*}\right)$ is connected.

Proof. In the notation of [7,

$$
\chi_{\left(s^{*}\right)}:=\left|W^{\circ}\left(s^{*}\right)\right|^{-1} \sum_{w \in W^{\circ}\left(s^{*}\right)} \varepsilon_{\mathbf{G}} \varepsilon_{\mathbf{T}_{w}^{*}} R_{\mathbf{T}_{w}^{*}}\left(s^{*}\right) .
$$

We begin by explaining this notation. First, $W^{\circ}\left(s^{*}\right)$ is the Weyl group of $C_{\mathbf{G}^{*}}^{\circ}\left(s^{*}\right)$, the connected component of $C_{\mathbf{G}^{*}}\left(s^{*}\right)$. Since $C_{\mathbf{G}^{*}}\left(s^{*}\right)$ is connected, we have $C_{\mathbf{G}^{*}}^{\circ}\left(s^{*}\right)$ $=C_{\mathbf{G}^{*}}\left(s^{*}\right)$ and hence $W^{\circ}\left(s^{*}\right)=W\left(s^{*}\right)$ (see [7, Remark 2.4]). Second, $R_{\mathbf{T}_{w}^{*}}\left(s^{*}\right)$ denotes a Deligne-Lusztig character of $G$ of the form $R_{\mathbf{T}, \vartheta}$, where $(\mathbf{T}, \vartheta) \in \mathcal{T}(\mathbf{G})$ is dual to $\left(\mathbf{T}_{w}^{*}, s^{*}\right) \in \mathcal{S}\left(\mathbf{G}^{*}\right)$, and where $\mathbf{T}_{w}^{*}$ is obtained from the reference torus of $C_{\mathbf{G}^{*}}\left(s^{*}\right)$ by twisting with $w$ (cf. Subsection 2.2).

Let $\kappa^{*}\left(s^{*}\right)$ denote a set of representatives for the $F^{*}$-conjugacy classes of $W\left(s^{*}\right)$. Then, again by the results summarized in Subsection 2.2 , we have

$$
\chi_{\left(s^{*}\right)}=\sum_{w \in \kappa^{*}\left(s^{*}\right)} \frac{\varepsilon_{\mathbf{G}} \varepsilon_{\mathbf{T}_{w}^{*}}}{\left|C_{W\left(s^{*}\right), F^{*}}(w)\right|} R_{\mathbf{T}_{w}^{*}}\left(s^{*}\right) .
$$

Every element of $\kappa\left(s^{*}\right)$ is dual (in the sense of (7) ) to a pair $\left(\mathbf{T}^{\prime *}, s^{*}\right) \in \mathcal{S}\left(\mathbf{G}^{*}\right)$; since $s^{*} \in \mathbf{T}^{\prime *}$, we have in fact $\left(\mathbf{T}^{\prime *}, s^{*}\right) \in \mathcal{S}\left(C_{\mathbf{G}^{*}}\left(s^{*}\right)\right)$. Two such pairs are conjugate in $G^{*}$ if and only if they are conjugate in $C_{\mathbf{G}^{*}}\left(s^{*}\right)^{F^{*}}$. Thus there is a bijection $\kappa^{*}\left(s^{*}\right) \rightarrow \kappa\left(s^{*}\right)$ such that $(\mathbf{T}, \vartheta) \in \kappa\left(s^{*}\right)$ is dual to $\left(\mathbf{T}_{w}^{*}, s^{*}\right)$ if $w \in \kappa^{*}\left(s^{*}\right)$ is mapped to $(\mathbf{T}, \vartheta)$. By Lemma 3.2 we have $\left|W(\mathbf{T})_{\theta}^{F}\right|=\left|W\left(\mathbf{T}_{w}^{*}\right)_{s^{*}}^{F^{*}}\right|$ for pairs corresponding this way.

Note that $W\left(\mathbf{T}_{w}^{*}\right)_{s^{*}}^{F^{*}}=N_{C_{\mathbf{G}\left(s^{*}\right)}}\left(\mathbf{T}_{w}^{*}\right)^{F^{*}} / \mathbf{T}_{w}^{* F^{*}} \cong C_{W\left(s^{*}\right), F^{*}}(w)$, the latter by [6, Proposition 3.3.6], applied to $C_{\mathbf{G}^{*}}\left(s^{*}\right)$. This completes the proof.

The above result does not hold if $C_{\mathbf{G}^{*}}\left(s^{*}\right)$ is not connected. Consider, for example, the case $\mathbf{G}=\operatorname{Sp}(2, \mathbf{K}) \cong \operatorname{SL}(2, \mathbf{K})$, where $q$ is odd. There is an involution $s^{*} \in G^{*}=\operatorname{SO}(3, q) \cong \operatorname{PGL}(2, q)$ whose centralizer is equal to $N_{\mathbf{G}^{*}}\left(\mathbf{T}_{0}^{*}\right)$. If $\left(\mathbf{T}_{\mathbf{0}}, \theta\right)$ is dual to $\left(\mathbf{T}_{0}^{*}, s^{*}\right)$, then $\theta=1_{T_{0}}^{-}$, and $\left|W\left(\mathbf{T}_{0}\right)_{\theta}^{F}\right|=2$. Since $\left|W^{\circ}\left(s^{*}\right)\right|=1$, we have $\chi_{\left(s^{*}\right)}=2 \rho_{s^{*}}$.

For the sake of uniform notation, we introduce a basis $v_{1}^{*}, v_{2}^{*}, \ldots, v_{n}^{*},\left[v_{0}^{*},\right]$ $v_{n}^{* \prime}, \ldots, v_{2}^{* \prime}, v_{1}^{* \prime}$ of the vector space $V^{*}$ (where $v_{0}^{*}$ is not present if $d=\operatorname{dim} V^{*}$ 
is even), such that $v_{1}^{*}, v_{2}^{*}, \ldots, v_{n}^{*}$ and $v_{n}^{* \prime}, \ldots, v_{2}^{* \prime}, v_{1}^{* \prime}$ span maximal isotropic subspaces of $V^{*}$ and the Hermitean or orthogonal form takes value 1 on the pairs $v_{i}^{*}, v_{i}^{* \prime}, 1 \leq i \leq n$, and $v_{0}^{*}$, if present, has norm 1. (Thus in the orthogonal case we have just "starred" the basis from 2.4)

Lemma 5.2. Let $s^{*}$ be a semisimple element of $G^{*}$ without eigenvalue $(-1)^{q}$ on $V^{*}$. Then $\pi_{s^{*}}=\rho_{s^{*}} \in \operatorname{Irr}(G)$.

Proof. We have $\left(\omega_{T}, \theta\right)=1$ for all $(\mathbf{T}, \theta) \in \kappa\left(s^{*}\right)$ by Lemmas 4.14(3) and 3.2(b). Hence the expression for $\pi_{s^{*}}$ coincides with that for $\rho_{s^{*}}$.

Now $C_{\mathbf{G}^{*}}\left(s^{*}\right)$ is connected since $s^{*}$ does not have eigenvalue $(-1)^{q}$. (If $\mathbf{G}^{*}=$ $\mathrm{GL}_{n}(\mathbf{K})$, the centralizer of every semisimple element is connected. In the other case, the result can be derived from [6, Theorem 3.5.3].) The irreducibility of $\rho_{s^{*}}$ follows from Lusztig's results in [15] (see also [7, 14.40, 14.43, 14.48] in connection with Lemma 5.1).

Our goal now is to determine the class functions $\pi_{s^{*}}$ in case $s^{*}$ has eigenvalue $(-1)^{q}$ on $V^{*}$.

Lemma 5.3. Let $s^{*} \in G^{*}$ be a semisimple element which has eigenvalue $(-1)^{q}$ on $V^{*}$ and suppose that $\pi_{s^{*}} \neq 0$. Then $s^{*}$ is conjugate in $\mathbf{G}^{*}$ to an element whose $(-1)^{q}$-eigenspace on $V^{*}$ equals $\left\langle v_{1}^{*}, \ldots, v_{m}^{*}, v_{m}^{*}{ }^{\prime}, \ldots, v_{1}^{* \prime}\right\rangle$ for some $1 \leq m \leq n$.

Proof. Denote by $V_{-}^{*}$ the $(-1)^{q}$-eigenspace of $s^{*}$, and by $\left(V_{-}^{*}\right)^{\perp}$ its orthogonal complement. Every element of $G^{*}$ commuting with $s^{*}$ fixes $V_{-}^{*}$ and $\left(V_{-}^{*}\right)^{\perp}$, and so every maximal torus $T^{*}$ of $G^{*}$ containing $s^{*}$ yields a $T^{*}$-decomposition of $V^{*}$ compatible with the direct sum $V^{*}=V_{-}^{*} \oplus\left(V_{-}^{*}\right)^{\perp}$.

Let $T^{*}$ be a maximal torus of $G^{*}$ with $s^{*} \in T^{*}$ and let $V^{*}=V_{0}^{*} \oplus V_{1}^{*} \oplus \cdots \oplus$ $V_{k}^{*} \oplus V_{k+1}^{*} \oplus \cdots \oplus V_{k+l}^{*}$ be such a compatible $T^{*}$-decomposition.

Suppose first that $\left(\mathbf{G}^{*}, F^{*}\right)$ is unitary, and that $\operatorname{dim} V_{-}^{*}$ is odd. Then $V_{j}^{*} \subseteq V_{-}^{*}$ for some $j>k$ by Lemma 2.2(1). Now let $\left(\mathbf{G}^{*}, F^{*}\right)$ be orthogonal. Then $V_{-}^{*}$ has even dimension $2 m$. Suppose that the Witt index of $V_{-}^{*}$ is smaller than $m$. Then, again, $V_{j}^{*} \subseteq V_{-}^{*}$ for some $j>k$ by Lemma 2.2(2). It follows that in the decomposition of the corresponding pair $(T, \theta)$, we have $\theta_{j}=1_{T_{j}}^{-}$by Lemma 3.2. By Lemma 4.14 $(1)$ this implies that $\left(\omega_{T}, \theta\right)=0$. Thus $\pi_{s^{*}}=0$ contrary to our assumption.

Hence $\operatorname{dim} V_{-}^{*}=2 m$ is even, and the Witt index of $V_{-}^{*}$ equals $m$ in the orthogonal case. By Witt's theorem we may assume that $V_{-}^{*}=\left\langle v_{1}^{*}, \ldots, v_{m}^{*}, v_{m}^{* \prime}, \ldots, v_{1}^{* \prime}\right\rangle$.

For $1 \leq m \leq n$ write

$$
\mathbf{V}^{(m)^{*}}:=\left\langle v_{1}^{*}, \ldots, v_{m}^{*}, v_{m}^{* \prime}, \ldots, v_{1}^{* \prime}\right\rangle_{\mathbf{K}}
$$

and

$$
\mathbf{V}^{\left(m^{\prime}\right)^{*}}:=\left\langle v_{m+1}^{*}, \ldots, v_{n}^{*},\left[v_{0}^{*},\right] v_{n}^{* \prime}, \ldots, v_{m+1}^{*}{ }^{\prime}\right\rangle_{\mathbf{K}},
$$

where the notation $\left[v_{0}^{*}\right]$ indicates that $v_{0}^{*}$ is to be omitted if $\operatorname{dim} \mathbf{V}^{*}$ is even. As usual we denote the sets of rational points of these vector spaces by $V^{(m)^{*}}$ and $V^{\left(m^{\prime}\right)^{*}}$, respectively. Then $V^{\left(m^{\prime}\right)^{*}}$ is the orthogonal complement of $V^{(m)^{*}}$.

Let $\mathbf{G}^{(m)^{*}}$ denote the subgroup of $\mathbf{G}^{*}$ fixing $\mathbf{V}^{(m)^{*}}$ and acting as the identity on $\mathbf{V}^{\left(m^{\prime}\right)^{*}}$, and let $\mathbf{G}^{\left(m^{\prime}\right)^{*}}$ be defined similarly. Then $\mathbf{G}^{(m)^{*}} \times \mathbf{G}^{\left(m^{\prime}\right)^{*}} \leq \mathbf{G}^{*}$ is the identity component of the stabilizer in $\mathbf{G}^{*}$ of the direct sum decomposition $\mathbf{V}^{*}=\mathbf{V}^{(m)^{*}} \oplus \mathbf{V}^{\left(m^{\prime}\right)^{*}}$. 
Lemma 5.4. Fix $1 \leq m \leq n$ and let $s^{*} \in G^{*}$ be a semisimple element whose $(-1)^{q}$-eigenspace on $V^{*}$ equals $V^{(m)^{*}}=\left\langle v_{1}^{*}, \ldots, v_{m}^{*}, v_{m}^{*}{ }^{\prime}, \ldots, v_{1}^{* \prime}\right\rangle$.

Let $\mathbf{T}^{*}$ be an $F^{*}$-stable maximal torus of $\mathbf{G}^{*}$ containing $s^{*}$ and let $(\mathbf{T}, \theta) \in \mathcal{T}(\mathbf{G})$ be dual to $\left(\mathbf{T}^{*}, s^{*}\right)$. If $\left(\omega_{T}, \theta\right) \neq 0$, then $\mathbf{T}^{*}$ is conjugate in $G^{*}$ to a torus fixing $\left\langle v_{1}^{*}, \ldots, v_{m}^{*}\right\rangle_{\mathbf{K}}$.

Proof. Clearly, $\mathbf{T}^{*}$ fixes the $(-1)^{q}$-eigenspace $\mathbf{V}^{(m)^{*}}$ of $s^{*}$ and its orthogonal complement $\mathbf{V}^{\left(m^{\prime}\right)^{*}}$. Consider a $T^{*}$-decomposition $V^{*}=V_{0}^{*} \oplus V_{1}^{*} \oplus \cdots \oplus V_{k}^{*} \oplus V_{k+1}^{*} \oplus \cdots \oplus$ $V_{k+l}^{*}$ of $V^{*}$ compatible with the orthogonal decomposition $V^{*}=V^{(m)^{*}} \oplus V^{\left(m^{\prime}\right)^{*}}$. If $V_{j}^{*} \leq V^{(m)^{*}}$ for some $j>k$, then, in the decomposition of the corresponding pair $(T, \theta)$, we have $\theta_{j}=1_{T_{j}}^{-}$by Lemma 3.2, and so $\left(\omega_{T}, \theta\right)=0$ by Lemma 4.14(1). Thus our assumption implies that $V^{(m)^{*}}$ is a direct sum of some $V_{j}^{*}$ s with $1 \leq j \leq k$, and so $T^{*}$ fixes a maximal singular subspace of $V^{(m)^{*}}$. By conjugating $\mathbf{T}^{*}$ by an element of $G^{*}$, we may assume that $T^{*}$ fixes $\left\langle v_{1}^{*}, \ldots, v_{m}^{*}\right\rangle$.

If $\operatorname{dim} \mathbf{V}^{*}$ is odd, we may also assume that $\mathbf{T}^{*}$ fixes $v_{0}^{*}$, by conjugating $\mathbf{T}^{*}$ with a suitable element of $G^{\left(m^{\prime}\right)^{*}}$. It follows that $\mathbf{T}^{*}$ fixes the space $\left\langle v_{0}^{*}\right\rangle_{\mathbf{K}} \oplus \mathbf{V}^{(m)^{*}}$ in this case. We may thus assume that $m=n$. Using the classification of the maximal tori in Sections 2.3 and 2.4 , we see that $\mathbf{T}^{*}$ is conjugate in $G^{*}$ to a maximal $F^{*}$ stable torus fixing $\left\langle v_{1}^{*}, \ldots, v_{n}^{*}\right\rangle_{\mathbf{K}}$. (If this were not the case, then $T^{*}$ would have an irreducible direct summand different from $\left\langle v_{0}^{*}\right\rangle$ in a $T^{*}$-decomposition of $V^{*}$.)

Thus we may assume that every pair $(\mathbf{T}, \theta)$ which contributes a non-zero summand to the sum (9) is dual to a pair $\left(\mathbf{T}^{*}, s^{*}\right)$ such that $\mathbf{T}^{*}$ fixes $\left\langle v_{1}^{*}, \ldots, v_{m}^{*}\right\rangle_{\mathbf{K}}$ for some $1 \leq m \leq n$. In other words, $\mathbf{T}^{*}$ lies in the standard (split) Levi subgroup $\mathbf{L}^{(m)^{*}} \times \mathbf{G}^{\left(m^{\prime}\right)^{*}}$ of $\mathbf{G}^{*}$ fixing $\left\langle v_{1}^{*}, \ldots, v_{m}^{*}\right\rangle_{\mathbf{K}}$. Here, $\mathbf{L}^{(m)^{*}}$ denotes the standard Levi subgroup of $\mathbf{G}^{(m)^{*}}$ fixing $\left\langle v_{1}^{*}, \ldots, v_{m}^{*}\right\rangle_{\mathbf{K}}$. Moreover, two such tori are conjugate in $G^{*}$ if and only if they are conjugate in $L^{(m)^{*}} \times G^{\left(m^{\prime}\right)^{*}}$.

We now fix $1 \leq m \leq n$, an element $s^{*} \in G^{*}$ whose $(-1)^{q}$-eigenspace on $V^{*}$ equals $V^{(m)^{*}}$, and a maximal torus $\mathbf{T}^{*} \leq \mathbf{L}^{(m)^{*}} \times \mathbf{G}^{\left(m^{\prime}\right)^{*}}$ containing $s^{*}$. Let $(\mathbf{T}, \theta) \in \mathcal{T}(\mathbf{G})$ be a pair dual to $\left(\mathbf{T}^{*}, s^{*}\right)$. Since duality behaves well with respect to split Levi subgroups, we may assume that $\mathbf{T} \leq \mathbf{L}^{(m)} \times \mathbf{G}^{\left(m^{\prime}\right)}$, the standard Levi subgroup of $\mathbf{G}$ fixing the isotropic subspace $\left\langle v_{1}, \ldots, v_{m}\right\rangle_{\mathbf{K}}$ of $\mathbf{V}$.

We have $\mathbf{L}^{(m)} \cong \mathrm{GL}(m, \mathbf{K})$ (acting on $\left\langle v_{1}, \ldots, v_{m}\right\rangle_{\mathbf{K}}$ ). Furthermore, we may assume that $\mathbf{T}=\mathbf{T}^{(m)} \times \mathbf{T}^{\left(m^{\prime}\right)}$ with $F$-stable maximal tori of $\mathbf{L}^{(m)}$ and of $\mathbf{G}^{\left(m^{\prime}\right)}$, respectively, and we have a corresponding decomposition $\theta=\theta^{(m)} \otimes \theta^{\left(m^{\prime}\right)}$.

To simplify notation, we put $\mathbf{L}:=\mathbf{L}^{(m)}, \mathbf{G}^{\prime}:=\mathbf{G}^{\left(m^{\prime}\right)}, \mathbf{S}:=\mathbf{T}^{(m)}, \mathbf{T}^{\prime}:=\mathbf{T}^{\left(m^{\prime}\right)}$, $\sigma:=\theta^{(m)}$ and $\theta^{\prime}:=\theta^{\left(m^{\prime}\right)}$. Then $\mathbf{T}=\mathbf{S} \times \mathbf{T}^{\prime}$ and $\theta=\sigma \otimes \theta^{\prime}$.

Lemma 5.5. With the above notation we have:

$$
\frac{\left(\omega_{T}, \theta\right)}{\left|W(\mathbf{T})_{\theta}^{F}\right|}=\frac{1}{\left|W_{\mathbf{L}}(\mathbf{S})_{\sigma}^{F}\right|} \cdot \frac{1}{\left|W_{\mathbf{G}^{\prime}}\left(\mathbf{T}^{\prime}\right)_{\theta^{\prime}}^{F}\right|}
$$

Proof. Let $\mathbf{H}$ denote the subgroup of $\mathbf{G}$ fixing $\mathbf{V}^{(m)}$ and acting as the identity on its complement $\mathbf{V}^{\left(m^{\prime}\right)}$. Then $\mathbf{H}$ is a general linear or symplectic group of dimension $2 m$ over $\mathbf{K}$. Moreover, $S$ is a neutral maximal torus of $H$. 
Using the multiplicity of the Weil representation (see Section 4) and Lemma 3.1 we find

$$
\frac{\left(\omega_{T}, \theta\right)}{\left|W(\mathbf{T})_{\theta}^{F}\right|}=\frac{\left(\omega_{S}^{(H)}, \sigma\right)}{\left|W_{\mathbf{H}}(\mathbf{S})_{\sigma}^{F}\right|} \cdot \frac{\left(\omega_{T^{\prime}}^{\left(G^{\prime}\right)}, \theta^{\prime}\right)}{\left|W_{\mathbf{G}^{\prime}}\left(\mathbf{T}^{\prime}\right)_{\theta^{\prime}}^{F}\right|}
$$

Now $\left(\omega_{T^{\prime}}^{\left(G^{\prime}\right)}, \theta^{\prime}\right)=1$ by Lemma 4.14(3). The claim follows as long as we can show that

$$
\frac{\left(\omega_{S}^{(H)}, \sigma\right)}{\left|W_{\mathbf{H}}(\mathbf{S})_{\sigma}^{F}\right|}=\frac{1}{\left|W_{\mathbf{L}}(\mathbf{S})_{\sigma}^{F}\right|}
$$

Let $\left(1^{l_{1}}, 2^{l_{2}}, \ldots, m^{l_{m}}\right)$ be the partition of $m$ defining the neutral maximal torus $\mathbf{S}$ of $\mathbf{H}$ (see Lemma 2.2 $(3))$. By Lemma 4.14 $(2),\left(\omega_{S}^{(H)}, \sigma\right)=2^{k(\sigma)}=2^{l_{1}+\cdots+l_{m}}$. By Lemma 2.3. $\left|W_{\mathbf{H}}(\mathbf{S})^{F}\right|=2^{l_{1}+\cdots+l_{m}}\left|W_{\mathbf{L}}(\mathbf{S})^{F}\right|$, proving the desired result.

We return to the computation of $\pi_{s^{*}}$, with $s^{*}$ as above. Write $s^{*}=(-1)^{q} \times s^{* \prime}$ with $(-1)^{q} \in L^{(m)^{*}}$ and $s^{*^{\prime}} \in G^{\left(m^{\prime}\right)^{*}}$. By the considerations above, if $(\mathbf{T}, \theta) \in$ $\mathcal{T}(\mathbf{G})$ is dual to $\left(\mathbf{T}^{*}, s^{*}\right)$, and if $\left(\omega_{T}, \theta\right) \neq 0$, then we may assume that there is a factorisation

$$
(\mathbf{T}, \theta)=\left(\mathbf{S} \times \mathbf{T}^{\prime}, \sigma \otimes \theta^{\prime}\right),
$$

in such a way that $(\mathbf{S}, \sigma) \in \mathcal{T}(\mathbf{S})$ is dual to $\left(\mathbf{S}^{*},(-1)^{q}\right) \in \mathcal{S}\left(\mathbf{L}^{*}\right)$ and $\left(\mathbf{T}^{\prime}, \theta^{\prime}\right) \in$ $\mathcal{T}\left(\mathbf{G}^{\prime}\right)$ is dual to $\left(\mathbf{T}^{\prime *}, s^{* \prime}\right) \in \mathcal{S}\left(\mathbf{G}^{\prime *}\right)$. Thus we may restrict summation in (9) to $\kappa_{\mathbf{L} \times \mathbf{G}^{\prime}}\left(s^{*}\right)=\kappa_{\mathbf{L}}\left((-1)^{q}\right) \times \kappa_{\mathbf{G}^{\prime}}\left(s^{* \prime}\right)$, with the obvious interpretation of $\kappa_{\mathbf{L}}$ and $\kappa_{\mathbf{G}^{\prime}}$.

Let $\mathbf{P}$ denote the standard parabolic subgroup of $\mathbf{G}$ fixing the isotropic subspace $\left\langle v_{1}, \ldots, v_{m}\right\rangle_{\mathbf{K}}$ of $\mathbf{V}$. By [6, Proposition 7.4.4], $R_{\mathbf{T}, \theta}^{\mathbf{G}}=\left(\operatorname{Infl}_{P}\left(R_{\mathbf{T}, \theta}^{\mathbf{L} \times \mathbf{G}^{\prime}}\right)\right)^{G}$ where $\operatorname{Infl}_{P}(\psi)$ denotes the inflation of the class function $\psi$ of $L \times G^{\prime}$ to $P$ via the homomorphism $P \rightarrow L \times G^{\prime}$.

Lemma 5.6. Let the notation be as above. Since $s^{*} \leq \mathbf{L}^{*} \times \mathbf{G}^{\prime *}$, we have a class function $\rho_{s^{*}}^{\left(L \times G^{\prime}\right)}$ of $L \times G^{\prime}$ defined analogously to $\rho_{s^{*}}$ for $G$. With this notation we have $\pi_{s^{*}}=\left(\operatorname{Infl}_{P}\left(\rho_{s^{*}}^{\left(L \times G^{\prime}\right)}\right)\right)^{G}$. In addition, $\rho_{s^{*}}^{\left(L \times G^{\prime}\right)}=\mathrm{St}_{L}^{-} \otimes \rho_{s^{* \prime}}^{\left(G^{\prime}\right)}$, where $\mathrm{St}_{L}^{-}=$ $1_{L}^{\bar{L}} \cdot \mathrm{St}_{L}$.

Proof. We have

$$
\begin{aligned}
\pi_{s^{*}} & =\sum_{(\mathbf{T}, \theta) \in \kappa\left(s^{*}\right)} \frac{\varepsilon_{\mathbf{G}} \varepsilon_{\mathbf{T}}\left(\omega_{T}, \theta\right)}{\left|W(\mathbf{T})_{\theta}^{F}\right|} R_{\mathbf{T}, \theta}^{\mathbf{G}} \\
& =\left(\operatorname{Infl}_{P}\left(\sum_{(\mathbf{T}, \theta) \in \kappa_{\mathbf{L} \times \mathbf{G}^{\prime}}\left(s^{*}\right)} \frac{\varepsilon_{\mathbf{G}} \varepsilon_{\mathbf{T}}\left(\omega_{T}, \theta\right)}{\left|W(\mathbf{T})_{\theta}^{F}\right|} R_{\mathbf{T}, \theta}^{\mathbf{L} \times \mathbf{G}^{\prime}}\right)\right)^{G} .
\end{aligned}
$$

By Lemma 5.5 and the discussion above, we find

$$
\begin{aligned}
& \sum_{(\mathbf{T}, \theta) \in \kappa_{\mathbf{L} \times \mathbf{G}^{\prime}}\left(s^{*}\right)} \frac{\varepsilon_{\mathbf{G}} \varepsilon_{\mathbf{T}}\left(\omega_{T}, \theta\right)}{\left|W(\mathbf{T})_{\theta}^{F}\right|} R_{\mathbf{T}, \theta}^{\mathbf{L} \times \mathbf{G}^{\prime}} \\
= & \sum_{(\mathbf{S}, \sigma) \in \kappa_{\mathbf{L}}\left((-1)^{q}\right)\left(\mathbf{T}^{\prime}, \theta^{\prime}\right) \in \kappa_{\mathbf{G}^{\prime}\left(s^{* \prime}\right)}} \frac{\varepsilon_{\mathbf{L}} \varepsilon_{\mathbf{S}}}{\left|W_{\mathbf{L}}(\mathbf{S})_{\sigma}^{F}\right|} \cdot \frac{\varepsilon_{\mathbf{G}^{\prime}} \varepsilon_{\mathbf{T}^{\prime}}}{\left|W_{\mathbf{G}^{\prime}}\left(\mathbf{T}^{\prime}\right)_{\theta^{\prime}}^{F}\right|} R_{\mathbf{T}, \sigma \otimes \theta^{\prime}}^{\mathbf{L} \times \mathbf{G}^{\prime}}
\end{aligned}
$$


Observe that $R_{\mathbf{T}, \sigma \otimes \mathbf{G}^{\prime}}^{\mathbf{L} \times \mathbf{G}^{\prime}}=R_{\mathbf{S}, \sigma}^{\mathbf{L}} \otimes R_{\mathbf{T}^{\prime}, \theta^{\prime}}^{\mathbf{G}^{\prime}}$. Therefore, the right-hand side of the above expression equals the product

$$
\left(\sum_{(\mathbf{S}, \sigma) \in \kappa_{\mathbf{L}}\left((-1)^{q}\right)} \frac{\varepsilon_{\mathbf{L}} \varepsilon_{\mathbf{S}}}{\left|W_{\mathbf{L}}(\mathbf{S})_{\sigma}^{F}\right|} R_{\mathbf{S}, \sigma}^{\mathbf{L}}\right) \otimes\left(\sum_{\left(\mathbf{T}^{\prime}, \theta^{\prime}\right) \in \kappa_{\mathbf{G}^{\prime}}\left(s^{* \prime}\right)} \frac{\varepsilon_{\mathbf{G}^{\prime}} \varepsilon_{\mathbf{T}^{\prime}}}{\left|W_{\mathbf{G}^{\prime}}\left(\mathbf{T}^{\prime}\right)_{\theta^{\prime}}^{F}\right|} R_{\mathbf{T}^{\prime}, \theta^{\prime}}^{\mathbf{G}^{\prime}}\right) .
$$

We have $\sigma=1_{S}^{-}$for all pairs $(\mathbf{S}, \sigma)$ occurring in the above sum. Hence $R_{\mathbf{S}, \sigma}^{\mathbf{L}}=$ $1_{L}^{-} \cdot R_{\mathbf{S}, 1_{S}}^{\mathbf{L}}$ and $W_{\mathbf{L}}(\mathbf{S})_{\sigma}^{F}=W_{\mathbf{L}}(\mathbf{S})_{1_{S}}^{F}$ for all such pairs. It follows that the first of these factors equals $\mathrm{St}_{L}^{-}$(see [6, Corollary 7.6.6]), while the second one, by definition, is equal to $\rho_{s^{\prime *}}^{\left(G^{\prime}\right)}$. Note that the latter is an irreducible character by Lemma 5.2 .

Proof of Theorem 1.2 (for symplectic and unitary groups). Set $\gamma=\sum_{\left(s^{*}\right) \in S^{*}} \rho_{s^{*}}$. If $Z(\mathbf{G})$ is connected, then $\gamma$ is known to coincide with the Gelfand-Graev character of $G$. Denote by $\gamma^{\prime}$ the "truncated" character obtained from $\gamma$ by removing all $\rho_{s^{*}}$ with $s^{*}$ having eigenvalue $(-1)^{q}$.

Now (8), (10), Lemma 5.2, and Lemma 5.6 yield a proof of Theorem 1.2 for the symplectic and unitary groups.

Proof of Corollary 1.3. If $\left(s_{1}^{*}\right)$ and $\left(s_{2}^{*}\right)$ are distinct elements of $S^{*}$, the constituents of $\pi_{s_{1}^{*}}$ and $\pi_{s_{2}^{*}}$ lie in distinct Lusztig series of characters. Hence it suffices to show that $\pi_{s^{*}}$ is multiplicity free, if $s^{*}$ has a $2 m$-dimensional $(-1)^{q}$-eigenspace for some $1 \leq m \leq n$.

Lemma 5.6 shows that $\pi_{s^{*}}=\left(\operatorname{Infl}_{P}\left(\mathrm{St}_{L}^{-} \otimes \rho_{s^{* \prime}}^{\left(G^{\prime}\right)}\right)\right)^{G}$. We may use Harish-Chandra theory to see that this Harish-Chandra induced character is multiplicity free. If $D$ denotes the maximally split torus of $L$, then clearly $\mathrm{St}_{L}^{-}$lies in the $\left(D, 1_{D}^{-}\right)$HarishChandra series of $L$. Let $M^{\prime}$ be a Levi subgroup of $G^{\prime}$ and $\tau^{\prime}$ an irreducible cuspidal character of $M^{\prime}$ such that $\rho_{s^{* \prime}}^{\left(G^{\prime}\right)}$ lies in the Harish-Chandra $\left(M^{\prime}, \tau^{\prime}\right)$-series of $G^{\prime}$. Then all constituents of $\pi_{s^{*}}$ and the irreducible character $\operatorname{St}_{L}^{-} \otimes \rho_{s^{*}}^{\left(G^{\prime}\right)}$ lie in the $\left(D \times M^{\prime}, 1_{D}^{-} \otimes \tau^{\prime}\right)$ Harish-Chandra series of $G$ and of $L \times G^{\prime}$, respectively.

In this proof we will use a slightly simplified notation for inertia factor groups of characters. If $\mathbf{M}$ is an $F$-stable Levi subgroup of $\mathbf{G}$ and $\eta$ an irreducible character of $M=\mathbf{M}^{F}$, we write $W_{G}(M)$ for the relative Weyl group $N_{\mathbf{G}}(\mathbf{M})^{F} / \mathbf{M}^{F}$ and $W_{G}(M, \eta)$ for the stabilizer of $\eta$ in $W_{G}(M)$.

It is well known that Harish-Chandra induction preserves Lusztig series. We may thus assume that $s^{* \prime}$ is contained in $M^{\prime *}$ and that $\tau^{\prime}$ lies in the Lusztig $s^{* \prime}$-series of $\operatorname{Irr}\left(M^{\prime}\right)$. One observes that $M^{\prime}$ is a direct product of $G L_{k_{i}}(q)$ (respectively $G L_{k_{i}}\left(q^{2}\right)$ if $G$ is unitary) and one factor of the same type as $G$. Correspondingly, $\tau^{\prime}$ is an outer product of irreducible cuspidal characters, one for each of the above factors of $M^{\prime}$. The group $W_{G}\left(D \times M^{\prime}, 1_{D}^{-} \times \tau^{\prime}\right)$ only permutes the factors of $M^{\prime}$ among themselves, since all factors of $\tau^{\prime}$ corresponding to a torus $G L_{1}(q)$ (respectively $G L_{1}\left(q^{2}\right)$ ) are not equal to $1^{-}$as $s^{* \prime}$ does not have eigenvalue $(-1)^{q}$. We thus find $W_{G}(D \times$ $\left.M^{\prime}, 1_{D}^{-} \otimes \tau^{\prime}\right)=W_{H}\left(D, 1_{D}^{-}\right) \times W_{G^{\prime}}\left(M^{\prime}, \tau^{\prime}\right)$, where $H$ has the same meaning as in the proof of Lemma 5.5. Clearly, $W_{L \times G^{\prime}}\left(D \times M^{\prime}, 1_{D}^{-} \otimes \tau^{\prime}\right)=W_{L}\left(D, 1_{D}^{-}\right) \times W_{G^{\prime}}\left(M^{\prime}, \tau^{\prime}\right)$. Now $W_{H}:=W_{H}\left(D, 1_{D}^{-}\right)$and $W_{L}:=W_{L}\left(D, 1_{D}^{-}\right)$are the Weyl groups of $H$ and $L$, respectively. The former is of type $B_{m}$, the latter is its parabolic subgroup of type $A_{m-1}$, obtained by deleting the outer node on the double bond of the Dynkin diagram. Via Harish-Chandra theory, the character $\mathrm{St}_{L}^{-} \otimes \rho_{s^{* \prime}}^{\left(G^{\prime}\right)}$ corresponds to a 
character $\operatorname{sgn} \bigotimes \lambda^{\prime}$, where sgn is the sign character of the symmetric group $W_{L} \cong S_{m}$ and $\lambda^{\prime}$ is some irreducible character of $W^{\prime}:=W_{G^{\prime}}\left(M^{\prime}, \tau^{\prime}\right)$.

By the comparison theorem of Howlett and Lehrer, the multiplicities of the irreducible constituents of $\left(\operatorname{Infl}_{P}\left(\mathrm{St}_{L}^{-} \otimes \rho_{s^{* \prime}}^{\left(G^{\prime}\right)}\right)\right)^{G}$ can be computed from the multiplicities in the induced character $\left(\operatorname{sgn} \otimes \lambda^{\prime}\right)_{W_{L} \times W^{\prime}}^{W_{H} \times W^{\prime}}$ (see [12, Theorem (5.9)]). We thus have to compute the constituents of $(\operatorname{sgn})_{W_{L}}^{W_{H}}$. This is the sum of all irreducible characters of $W_{H}$ which are labelled by bipartitions of $m$ whose parts are all equal to 1, a fact that can be derived from a special case of the Littlewood-Richardson rule (see, e.g., 10, Lemma 6.1.4].) This completes the proof.

\section{The Weil Representation of the General Linear group}

Here, we consider the tensor product of the Weil representation of the general linear group with its Steinberg representation.

Gérardin defined the Weil representation of $G:=\operatorname{GL}(n, q)$ as the permutation representation of $G$ on the vectors of the underlying vector space (see 11, Corollary 1.4]). According to Definition [1.1, let us write $\hat{\omega}$ for this permutation character and put $\omega:=1_{G}^{-} \cdot \hat{\omega}$. We will compute $\hat{\omega} \cdot$ St, from which the desired result follows.

In order to proceed, we describe the stabilizer in $G$ of a non-zero vector, and its characters. For inductive reasons, we treat $n$, the dimension of the underlying vector space, as a parameter. In particular, we write $G_{n}$ for $G$.

For a positive integer $n$ let $Q_{n-1}$ denote the following subgroup of $\operatorname{GL}(n, q)$ :

$$
Q_{n-1}=\left\{\left[\begin{array}{cc}
1 & v^{t} \\
0 & x
\end{array}\right] \mid v \in \mathbb{F}_{q}^{n-1}, x \in \operatorname{GL}(n-1, q)\right\} .
$$

(By convention, $Q_{0}$ is the trivial subgroup of $\mathrm{GL}_{1}(q)$.) Thus $Q_{n-1}$ is the affine group of degree $n-1$. We identify $Q_{n-1}$ with the semidirect product $V_{n-1} G_{n-1}$, where $V_{n-1}$ is the unipotent radical of $Q_{n-1}$, consisting of those matrices in (11) with $x=1$.

Suppose now that $n \geq 2$. Since $G_{n-1}$ acts transitively on the non-identity elements of $V_{n-1}$, there are two types of irreducible characters of $Q_{n-1}$. The first type consists of the characters of $G_{n-1}$, inflated to characters of $V_{n-1} G_{n-1}$. For the second type, we choose a particular element $\lambda \in \operatorname{Irr}\left(V_{n-1}\right)$ such that the stabilizer of $\lambda$ in $G_{n-1}$ equals $Q_{n-2}$. Then the irreducible characters of $V_{n-1} G_{n-1}$, which do not have $V_{n-1}$ in their kernel, are parametrized by the irreducible characters of $Q_{n-2}$. We write $\psi_{\mu}$ for an irreducible character of the second type with parameter $\mu \in \operatorname{Irr}\left(Q_{n-2}\right)$. Thus $\psi_{\mu}=(\hat{\lambda} \cdot \tilde{\mu})^{V_{n-1} G_{n-1}}$, where $\hat{\lambda}$ is a trivial extension of $\lambda$ to its stabilizer $V_{n-1} Q_{n-2}$, and $\tilde{\mu}:=\operatorname{Infl}_{V_{n-1} Q_{n-2}}(\mu)$ is the inflation of $\mu$ to this stabilizer.

We choose the irreducible character $\lambda$ of $V_{n-1}$ as follows. Let $U_{n}$ denote the group of upper triangular unipotent matrices in $G_{n}$. Choose a non-trivial homomorphism $\nu: \mathbb{F}_{q} \rightarrow \mathbb{C}^{*}$. Then let $\lambda \in \operatorname{Irr}\left(U_{n}\right)$ be defined by $\lambda(u)=\prod_{i=1}^{n-1} \nu\left(u_{i, i+1}\right)$ for $u=\left(u_{i j}\right) \in U_{n}$. Then $\lambda^{G_{n}}=\gamma_{n}$, the character of the Gelfand-Graev representation of $G_{n}$, (see [6, Section 8.1]). We also denote by the same letter the restriction of $\lambda$ to any subgroup of $U_{n}$, in particular, to the subgroup $V_{n-1}$.

With this notation we are now going to define, recursively on $n-1$ and $i$, $0 \leq i \leq n-1$, the level-i-Steinberg character $\sigma_{i}^{(n-1)}$ of $Q_{n-1}$. To begin with, $\sigma_{0}^{(0)}$ is the trivial character of the trivial group $Q_{0}$. For $n \geq 2$ and $i=0$, we let

$\sigma_{0}^{(n-1)}$ denote the inflation of $\mathrm{St}_{n-1}$ to $V_{n-1} G_{n-1}$, and call it the level-0-Steinberg 
character of $Q_{n-1}=V_{n-1} G_{n-1}$. For $i \geq 1$, the level-i-Steinberg character of $Q_{n-1}$ is defined by $\sigma_{i}^{(n-1)}:=\psi_{\mu}$ for $\mu=\sigma_{i-1}^{(n-2)}$.

With this notation we can state our first result. This is a special case of the results of [5, Chapter 5].

Proposition 6.1. For all $n \geq 1$, we have $\left(\mathrm{St}_{n}\right)_{Q_{n-1}}=\sum_{i=0}^{n-1} \sigma_{i}^{(n-1)}$.

Proof. It is clear, that among the constituents of $\left(\mathrm{St}_{n}\right)_{Q_{n-1}}$ of the first type, only the inflation of the Steinberg character $\mathrm{St}_{n-1}$ occurs, and this with multiplicity 1. The result is trivial for $n=1$. Suppose that $n \geq 2$ and let $\mu \in \operatorname{Irr}\left(Q_{n-2}\right)$. Using the facts that $V_{n-1} Q_{n-2} G_{n-1}=Q_{n-1}$ and $V_{n-1} Q_{n-2} \cap G_{n-1}=Q_{n-2}$, as well as $\left(\mathrm{St}_{n}\right)_{Q_{n-1}}=\left(\mathrm{St}_{n-1}\right)^{Q_{n-1}}$ (see [6. Proposition 6.3.3]), we compute

$$
\begin{aligned}
\left(\left(\mathrm{St}_{n}\right)_{Q_{n-1}}, \psi_{\mu}\right) & =\left(\left(\mathrm{St}_{n-1}\right)^{Q_{n-1}}, \psi_{\mu}\right) \\
& =\left(\left(\mathrm{St}_{n-1}\right)^{Q_{n-1}},(\hat{\lambda} \cdot \tilde{\mu})^{Q_{n-1}}\right) \\
& =\left(\mathrm{St}_{n-1},\left((\hat{\lambda} \cdot \tilde{\mu})^{Q_{n-1}}\right)_{G_{n-1}}\right) \\
& =\left(\mathrm{St}_{n-1},\left((\hat{\lambda} \cdot \tilde{\mu})_{V_{n-1} Q_{n-2} \cap G_{n-1}}\right)^{G_{n-1}}\right) \\
& =\left(\mathrm{St}_{n-1}, \mu^{G_{n-1}}\right) \\
& =\left(\left(\mathrm{St}_{n-1}\right)_{Q_{n-2}}, \mu\right) .
\end{aligned}
$$

By induction, $\left(\mathrm{St}_{n-1}\right)_{Q_{n-2}}=\sum_{i=0}^{n-2} \sigma_{i}^{(n-2)}$, and the result follows.

Let $\hat{\omega}:=\hat{\omega}_{n}$ denote the permutation character of $G_{n}$ on its natural vector space. Thus $\hat{\omega}_{n}=1_{G}+\left(1_{Q_{n-1}}\right)^{G}$. Hence $\hat{\omega}_{n} \cdot \mathrm{St}_{n}=\mathrm{St}_{n}+\left(\left(\mathrm{St}_{n}\right)_{Q_{n-1}}\right)^{G_{n}}$.

Recall that $\gamma_{n}$ denotes the Gelfand-Graev character of $G_{n}$. (For $n=1, \gamma_{1}$ equals the regular character of $G_{1}=\operatorname{GL}(1, q)$.) For $0 \leq m \leq n$, we let $P_{m}$ denote the standard parabolic subgroup of $G_{n}$ corresponding to the composition $(m, n-m)$ of $n$. The unipotent radical of $P_{m}$ is denoted by $U_{m, n-m}$. The Levi subgroup of $P_{m}$ is isomorphic to $G_{m} \times G_{n-m}$ (with the convention that $G_{0}$ denotes the trivial group).

Theorem 6.2. Let $n \geq 1$ and $G=\operatorname{GL}(n, q)$. Then

$$
\hat{\omega} \cdot \mathrm{St}=\sum_{m=0}^{n}\left(\operatorname{Infl}_{P_{m}}\left(\mathrm{St}_{m} \otimes \gamma_{n-m}\right)\right)^{G} .
$$

Proof. The summand for $m=n$ on the right-hand side equals $\mathrm{St}=\mathrm{St}_{n}$. So it suffices to prove that

$$
\left(1_{Q_{n-1}}\right)^{G} \cdot \mathrm{St}_{n}=\sum_{m=0}^{n-1}\left(\operatorname{Infl}_{P_{m}}\left(\operatorname{St}_{m} \otimes \gamma_{n-m}\right)\right)^{G} .
$$

Now $\left(1_{Q_{n-1}}\right)^{G} \cdot \mathrm{St}_{n}=\left(\left(\mathrm{St}_{n}\right)_{Q_{n-1}}\right)^{G}=\left(\sum_{i=0}^{n-1} \sigma_{i}^{(n-1)}\right)^{G}$ by Proposition 6.1 To complete the proof we show that

$$
\left(\sigma_{i}^{(n-1)}\right)^{G}=\left(\operatorname{Infl}_{P_{n-i-1}}\left(\operatorname{St}_{n-i-1} \otimes \gamma_{i+1}\right)\right)^{G}
$$

for all $0 \leq i \leq n-1$.

Let us start with the case $i=0$. Here, $Q_{n-1}$ is a normal subgroup of $P_{1}$, in fact, $Q_{n-1}=V_{n-1} G_{n-1}$ and $P_{1}=V_{n-1}\left(G_{1} \times G_{n-1}\right)$ (in fact, $\left.V_{n-1}=U_{1, n-1}\right)$. Hence 
$\left(\sigma_{0}^{(n-1)}\right)^{P_{1}}=\operatorname{Infl}_{V_{n-1}}\left(\rho_{G_{1}} \otimes \mathrm{St}_{n-1}\right)$. It follows that $\left(\sigma_{0}^{(n-1)}\right)^{G}=\left(\operatorname{Infl}_{P_{1}}\left(\gamma_{1} \otimes \operatorname{St}_{n-1}\right)\right)^{G}$, as claimed.

For $i \geq 1$ (and hence $n \geq 2$ ) consider the subgroup $H:=\left(U_{i+1} \times G_{n-i-1}\right) U_{i+1, n-i-1}$ of $P_{i+1}=\left(G_{i+1} \times G_{n-i-1}\right) U_{i+1, n-i-1}$ (recall that $U_{m}$ denotes the group of upper triangular unipotent matrices in $\left.G_{m}\right)$. Clearly, $H \leq Q_{n-1}$. We claim that $\left(\operatorname{Infl}_{H}\left(\lambda \otimes \mathrm{St}_{n-i-1}\right)\right)^{Q_{n-1}}=\sigma_{i}^{(n-1)}$.

Suppose that this claim has been proved. Then

$$
\begin{aligned}
\left(\sigma_{i}^{(n-1)}\right)^{G} & =\left(\operatorname{Infl}_{H}\left(\lambda \otimes \operatorname{St}_{n-i-1}\right)\right)^{G} \\
& =\left(\left(\operatorname{Infl}_{H}\left(\lambda \otimes \operatorname{St}_{n-i-1}\right)\right)^{P_{i+1}}\right)^{G} \\
& =\left(\operatorname{Infl}_{P_{i+1}}\left(\gamma_{i+1} \otimes \mathrm{St}_{n-i-1}\right)\right)^{G},
\end{aligned}
$$

giving the result.

It suffices to prove the above claim. First, observe that $\sigma_{i}^{(n-1)}(1)=\left(q^{n-1}-\right.$ 1) $\left(q^{n-2}-1\right) \cdots\left(q^{n-i}-1\right) \mathrm{St}_{n-i-1}(1)$, and that this number also equals the degree of the induced character $\left(\operatorname{Infl}_{H}\left(\lambda \otimes \mathrm{St}_{n-i-1}\right)\right)^{Q_{n-1}}$. By definition, $\sigma_{i}^{(n-1)}=(\hat{\lambda}$. $\tilde{\mu})^{Q_{n-1}}$ with $\tilde{\mu}=\operatorname{Infl}_{V_{n-1} Q_{n-2}}\left(\sigma_{i-1}^{(n-2)}\right)$. Since $i \geq 1$, we have $H \leq V_{n-1} Q_{n-2}$, and thus it suffices to show that $\hat{\lambda} \cdot \operatorname{Infl}_{V_{n-1} Q_{n-2}}\left(\sigma_{i-1}^{(n-2)}\right)$ is a constituent of $\operatorname{Infl}_{H}(\lambda \otimes$ $\left.\left.\mathrm{St}_{n-i-1}\right)\right)^{V_{n-1} Q_{n-2}}$. By Frobenius reciprocity, we are left to show that

$$
\left(\hat{\lambda} \cdot \operatorname{Infl}_{V_{n-1} Q_{n-2}}\left(\sigma_{i-1}^{(n-2)}\right)\right)_{H}
$$

contains $\operatorname{Infl}_{H}\left(\lambda \otimes \mathrm{St}_{n-i-1}\right)$ as a constituent. This is done by induction on $n$, the case $n=2$ being trivial.

Since $V_{n-1} \leq H \leq V_{n-1} Q_{n-2}$, we have $H=V_{n-1} K$ with $K=H \cap Q_{n-2}$. Now $H / V_{n-1} \cong K=\left(U_{i} \times G_{n-i-1}\right) U_{i, n-i-1}$, and, by induction, $\lambda \otimes \mathrm{St}_{n-i-1}$ is a constituent of the restriction of $\sigma_{i-1}^{(n-2)}$ to $K$ (where $\lambda$ is considered as a character of $U_{i}$ ). By the definition of $\lambda$ and of $\hat{\lambda}$ above, it follows that the restriction of $\hat{\lambda} \cdot \operatorname{Infl}_{V_{n-1} Q_{n-2}}\left(\sigma_{i-1}^{(n-2)}\right)$ to $H$ contains $\lambda \otimes \mathrm{St}_{n-i-1}$ as a constituent. This completes the proof.

Multiplying the expression for $\hat{\omega}$ in Theorem 6.2 by $1_{G}^{-}$, yields the statement in Theorem 1.2 for the general linear groups.

By this theorem, $\hat{\omega} \cdot \mathrm{St}$ is not multiplicity free, since every $\gamma_{n-m}$ contains $\mathrm{St}_{n-m}$ as a constituent, and $\operatorname{Infl}_{P_{m}}\left(\mathrm{St}_{m} \otimes \mathrm{St}_{n-m}\right)^{G}$ contains $\mathrm{St}_{G}$ as a constituent. (By [12, Theorem (5.9)], the latter assertion can be transformed to a statement in the symmetric group $S_{n}$, where it is obvious.)

\section{Applications}

In this section we prove Theorems 1.4 and 1.5

7.1. Restricting the Steinberg character. If $G=\operatorname{Sp}(2 n, q), q$ odd, and $P$ denotes the stabilizer of a line in the natural module of $G$, the characters of $P$ have been described recursively in [1. Rather than recalling the details of [1, we discuss the corresponding problem for the unitary groups, which reveals a new type of problem. Thus let $G=U(d, q)$ acting on the vector space $V$ equipped with the Hermitian form as in Subsection 2.3. Let $P$ be the stabilizer of an isotropic line of $V$. Let $U$ be the unipotent radical of $P$, and let $Z(U)$ denote the center of $U$. 
Additionally, let $L$ denote a Levi subgroup of $P$. Then $P=L U$ and $L=L^{\prime} \times A$ with $L^{\prime} \cong U(d-2, q)$ and $A \cong \mathrm{GL}\left(1, q^{2}\right)$.

There are three types of characters of $P$ :

Type (A): The characters trivial on $U$.

Type (B): The characters non-trivial on $U$, but trivial on $Z(U)$.

Type (C): The characters non-trivial on $Z(U)$.

It is slightly less technical to work with the group $P^{\prime}:=L^{\prime} U$, the stabilizer of an isotropic vector. Thus $P^{\prime}$ is a normal subgroup of $P$ with cyclic quotient generated by $A$. This fact can be used to extend the results below from $P^{\prime}$ to $P$. Of course, the above classification of the irreducible characters also holds for $P^{\prime}$.

Set $\bar{U}:=U / Z(U)$. Observe that $\bar{U}$ is an abelian group which can be viewed as the natural $\mathbb{F}_{q^{2}} L^{\prime}$-module (that is, $\mathbb{F}_{q^{2}}^{d-2}$ ). The group $\operatorname{Irr}(\bar{U})$ of irreducible characters of $\bar{U}$ is isomorphic to $\bar{U}$ as abelian groups and as $\mathbb{F}_{q^{2}} L^{\prime}$-modules. In particular, if $\lambda \in \operatorname{Irr}(U)$, then the stabilizer of $\lambda$ in $L^{\prime}$ (or the inertia group) coincides with the stabilizer in $L^{\prime}$ of some element $\bar{U}$. This simplifies the study of the inertia groups.

Let $\chi$ be an irreducible character of $P^{\prime}$ of Type $B$. By Clifford's theorem, there is a non-trivial irreducible character $\lambda$ of $\bar{U}$ such that $\chi$ is induced from an irreducible character $\mu$, say, of the stabilizer $P_{\lambda}^{\prime}$ of $\lambda$ in $P^{\prime}$.

So the first matter is to describe $P_{\lambda}^{\prime}$. It has been observed above that $P_{\lambda}^{\prime}=$ $U \operatorname{Stab}_{L^{\prime}}(\lambda)$ and the second group here coincides with the stabilizer of some nonzero vector $v \in \mathbb{F}_{q^{2}}^{d-2}$. As $\mathbb{F}_{q^{2}}^{d-2}$ is the natural $\mathbb{F}_{q^{2}} L^{\prime}$-module, this space possesses a unitary form, so the vector in question can be either isotropic or anisotropic. The group $L^{\prime} \cong U(d-2, q)$ acts transitively on the set of (non-zero) isotropic vectors and has $q-1$ orbits on the set of anisotropic vectors, so $L^{\prime}$ has exactly $q$ orbits on the non-zero vectors of $\mathbb{F}_{q^{2}}^{d-2}$. Since the stabilizers of proportional vectors are the same, we may assume that the representatives of the orbits of anisotropic vectors all have the same stabilizer. Thus, $\chi$ corresponds either to an isotropic or to an anisotropic vector. Depending on this, we say that $\chi$ is of Type $(B 1)$ or $(B 2)$.

According to this, $P_{\lambda}^{\prime} / U$ is isomorphic either to $P_{d-2}^{\prime}$, where $P_{d-2}^{\prime}$ is the stabilizer in $L^{\prime} \cong U(d-2, q)$ of an isotropic vector, thus defined analogously to $P^{\prime}$, or $P_{\lambda}^{\prime} / U$ is isomorphic to $U(d-3, q)$, the stabilizer of an anisotropic vector. Thus the irreducible characters of $P^{\prime}$ of Type (B1) are naturally labelled (bijectively) by $\operatorname{Irr}\left(P_{d-2}^{\prime}\right)$, and those of Type (B2) by $\operatorname{Irr}(U(d-3, q))$ (for more details see [1, 2.3.2]). The characters of Type (B) are invariant in $P^{\prime}$, so each of them has exactly $q-1$ extensions to $P^{\prime}$.

Let $\chi=\chi_{\mu}$ be an irreducible character of $P^{\prime}$ of Type (B), labelled by the irreducible character $\mu$ of $H \leq U(d-2, q)$, with $H=P_{d-2}^{\prime}$ or $U(d-3, q)$, respectively. As in [1, Section 3], we have

$$
\left(\mathrm{St}_{P^{\prime}}, \chi_{\mu}\right)=\left(\mathrm{St}_{H}^{\left(L^{\prime}\right)}, \mu\right) .
$$

We have a similar result as in [1] for characters of Type (C). These can be labelled by $\operatorname{Irr}\left(L^{\prime}\right)$, such that $\vartheta \in \operatorname{Irr}\left(L^{\prime}\right)$ determines exactly $q-1$ irreducible characters $\psi_{\vartheta}^{i}$ of Type (C), permuted transitively by the action of $P$. Thus every $\psi_{\vartheta}^{i}$ induces to an irreducible character $\psi_{\vartheta}$ of $P$ of Type (C), whose restriction to $P^{\prime}$ equals $\sum_{i=1}^{q-1} \psi_{\vartheta}^{i}$.

If $\chi$ is an irreducible character of this type labelled by the pair $(\vartheta, i)$ with $\vartheta \in$ $\operatorname{Irr}(U(d-2, q))$ and $1 \leq i \leq q-1$, we have

$$
\left(\mathrm{St}_{P^{\prime}}, \chi\right)=\left(\omega^{\prime} \cdot \mathrm{St}_{L^{\prime}}, \vartheta\right)
$$


where $\omega^{\prime}$ denotes the Weil character of $U(d-2, q)$. In particular, this multiplicity is independent of $i$ and can be computed by Theorem 1.2 ,

Proof of Theorem 1.4. If $G=\mathrm{GL}(n, q)$, the result follows from [5, Chapter 5]. An explicit version is given in Proposition 6.1.

Next let $G=U(d, q)$. Suppose first that $H^{\prime} \leq G$ is the stabilizer of an anisotropic vector. By the result of Brunat (see the appendix), $\mathrm{St}_{H^{\prime}}$ equals the product of the Steinberg character and the Weil character of $H^{\prime}$. Using Corollary 1.3, the result follows in this case.

Now suppose that $P^{\prime} \leq G$ is the stabilizer of a non-zero isotropic vector. Clearly, the only character of Type (A) contained in $\mathrm{St}_{P^{\prime}}$ equals $\mathrm{St}_{L^{\prime}}$, and it occurs with multiplicity 1. Now lets look at characters of Type (B). For characters of Type (B1) we use (12) and induction on $d$ (the case of $d=2$ being clear). For characters of Type (B2) we have to determine the restriction of the Steinberg character of $L^{\prime}=U(d-2, q)$ to its subgroup $L^{\prime \prime}=U(d-3, q)$. By what we have proved already, this restriction is multiplicity free. The assertion for characters of Type (C) follows from (13) together with Corollary 1.3 ,

Finally, let $G=\operatorname{Sp}(2 n, q)$, and let $P$ denote the stabilizer of a line $\langle v\rangle$. If $n \leq 3$, the result is already contained in 1 . In the general case it follows from 1. Corollary 3.3], together with Corollary [1.3. Now suppose that $P^{\prime} \leq P$ is the stabilizer of the vector $v$. For characters of Type 3 (notation from [1]), the claim easily follows from Clifford theory applied to the normal subgroup $P^{\prime}$ of $P$. For characters of Type 2 we could also use Clifford theory, but it is simpler to use exactly the same direct approach as in the unitary groups for characters of Type (B1).

This completes the proof of Theorem 1.4

7.2. The decomposition of a projective character. In order to prove Theorem 1.5, we continue our investigation of the generalized spinor representation of the symplectic groups in characteristic 2 begun in Subsection4.2. In particular, we use the notation summarized there. Moreover, we let $s t$ denote the Brauer reduction modulo 2 of the Steinberg representation of $G=\operatorname{Sp}(2 n, q)$, where $q$ is a power of 2 . Then st is a projective $\mathbb{F}_{q} G$-representation. Hence every representation of the form $s t \otimes \phi$ is also projective for every representation $\phi$ of $\mathbb{F}_{q} G$.

\subsubsection{The product $\sigma_{n} \otimes \sigma_{n}$ and the natural permutation module $\Pi_{n}$.}

Lemma 7.1. The multiplicity of every irreducible $\mathbb{F}_{2}$-representation $\tau$ of $\operatorname{Sp}(2 n, 2)$ in $\left(\phi_{\lambda_{n}} \otimes \phi_{\lambda_{n}}\right)_{\mathrm{Sp}(2 n, 2)}$ is equal to the multiplicity of $\tau$ in the permutation module $\Pi_{n}$ of $\operatorname{Sp}(2 n, 2)$ associated with the natural action of $\operatorname{Sp}(2 n, 2)$ on the vectors of its standard module $V$ (the zero vector is not excluded).

Proof. It suffices to show that the Brauer characters of the two modules coincide. The action of the image $\eta(\operatorname{ESp}(2 n, 2))$ of the extrasymplectic group on the set of matrices $\operatorname{Mat}\left(2^{n}, \mathbb{C}\right)$ by conjugation turns $\operatorname{Mat}\left(2^{n}, \mathbb{C}\right)$ into a $\mathbb{C E S p}(2 n, 2)$-module. Of course, this is exactly the module afforded by $\bar{\eta} \otimes \eta$. By Corollaries 4.10 and 4.13 , the character of this module at an odd order element $g \in \operatorname{ESp}(2 n, 2)$ is equal to $2^{N(V ; h)}$ where $h$ is the projection of $g$ into $\operatorname{Sp}(2 n, 2)$ and $N(V ; h)$ is the dimension of the 1-eigenspace of $h$ on $V$. Obviously, this coincides with the character of $h$ on $\Pi_{n}$. By Proposition 4.9, the Brauer reduction modulo 2 of $\eta$ equals $\left(\phi_{\lambda_{n}}\right)_{\mathrm{Sp}(2 n, 2)}$. Hence, by Corollary 4.10, the reduction modulo 2 of $\bar{\eta} \otimes \eta$ has the same Brauer character as $\left(\bar{\phi}_{\lambda_{n}} \otimes \phi_{\lambda_{n}}\right)_{\mathrm{Sp}(2 n, 2)}$ and this coincides with $\left(\phi_{\lambda_{n}} \otimes \phi_{\lambda_{n}}\right)_{\mathrm{Sp}(2 n, 2)}$ as $\eta$ 
is real. So the Brauer character of $\left(\phi_{\lambda_{n}} \otimes \phi_{\lambda_{n}}\right)_{\operatorname{Sp}(2 n, 2)}$ coincides with the Brauer character of the permutation module in question.

Observe that the natural permutation $\mathbb{F}_{q} \operatorname{Sp}\left(2 m, 2^{k}\right)$-module can be identified with the restriction of $\Pi_{m k}$ to $\operatorname{Sp}\left(2 m, 2^{k}\right)$, where $\Pi_{m k}$ is the natural permutation $\mathbb{F}_{2} \operatorname{Sp}(2 m k, 2)$-module.

Lemma 7.2. For $0 \leq i \leq 2 n$ let $V_{i}$ denote the $i$ th exterior power of $V$, the natural $\mathbb{F}_{2} \mathrm{SL}(2 n, 2)$-module ( $V_{0}$ is regarded as the trivial module). Let $\tau$ be an $\mathbb{F}_{2} \mathrm{Sp}(2 n, 2)$ composition factor of $\Pi_{n}$.

(1) Then $\tau$ is isomorphic to a composition factor of $\left(V_{i}\right)_{\operatorname{Sp}(2 n, 2)}$ for some $i \leq n$.

(2) If $\mu$ is a composition factor of $\Pi_{n}$ viewed as $\mathbb{F}_{q} \operatorname{Sp}(2 m, q)$-module, where $q=2^{k}$ and $n=m k$, then $\mu=\left(\phi_{\lambda}\right)_{\operatorname{Sp}(2 m, q)}$ for $\lambda=\sum_{i=0}^{k-1} 2^{i} \lambda_{j_{i}}$ with $j_{i} \in\{0, \ldots, n\}$. (Recall that the $\lambda_{i}$ are the fundamental weights for $i=1, \ldots, n$ and $\lambda_{0}=0$.)

(3) There is at most one composition factor in (2) occurring with multiplicity 1; this is $\left(\phi_{\lambda}\right)_{\operatorname{Sp}(2 m, q)}$ where $\lambda=\sum_{i=0}^{k-1} 2^{i} \lambda_{m}=(q-1) \lambda_{m}$.

Proof. (1) and (2) are proved in [18, Proposition 3.5]. To justify (3), consider $\Pi_{n}$ and $V_{i}$ as $\mathrm{SL}(2 n, 2)$-modules, and consider $V_{i}$ as $\mathbb{F}_{q} \mathrm{SL}(2 n, q)$-module. The composition factors of $\left(\Pi_{n}\right)_{\mathrm{SL}(2 n, 2)}$ are irreducible $\mathbb{F}_{2} \mathrm{SL}(2 n, 2)$-modules isomorphic to $\left(V_{i}\right)_{\mathrm{SL}(2 n, 2)}$ for $i=0, \ldots, 2 n-1$, where each factor occurs with multiplicity 1 except for the trivial one which occurs twice. (This is well known, but one may consult [18. Theorem 1.4], where the composition factors of the permutation module of $\mathrm{SL}(m, q)$ on the vectors of the natural module have been determined.) Therefore, the multiplicity of every composition factor in $\left(\Pi_{n}\right)_{\mathrm{Sp}(2 n, 2)}$ and in $\bigoplus_{i=0}^{2 n}\left(V_{i}\right)_{\operatorname{Sp}(2 n, 2)}$ coincide. It is well known that $V_{i}$ and $V_{2 n-i}$ are dual $\mathrm{SL}(2 n, 2)$-modules. Therefore, $\left(V_{i}\right)_{\mathrm{Sp}(2 n, 2)} \cong\left(V_{2 n-i}\right)_{\mathrm{Sp}(2 n, 2)}$. It follows that the irreducible constituents of multiplicity 1 can only occur in $\left(V_{n}\right)_{\mathrm{Sp}(2 n, 2)}$. Observe that $\left(V_{i}\right)_{\mathrm{Sp}(2 n, 2)}$ for $i \leq n$ contains a composition factor $W_{i}$ of highest weight $\lambda_{i}$. By (1) only $\left(W_{n}\right)_{\operatorname{Sp}(2 n, 2)}$ can occur in $\left(\Pi_{n}\right)_{\operatorname{Sp}(2 n, 2)}$ with multiplicity 1 . This completes the case $q=2$. In general, it follows from this that only irreducible constituents of $\left(W_{n}\right)_{\operatorname{Sp}(2 m, q)}$ can occur with multiplicity 1 . By Lemma 4.7, $\left(W_{n}\right)_{\mathrm{Sp}(2 m, q)}$ is irreducible and coincides with $\left(\phi_{\lambda}\right)_{\operatorname{Sp}(2 m, q)}$ where $\lambda$ is as in statement (3).

Remark 7.3. (1) In fact, the composition factor $\phi_{\lambda}$ in (3) occurs with multiplicity 1. This can be proved straightforwardly, but we will deduce it later from the proof of Theorem 1.5. Observe that Corollary 4.8 implies that the composition factors of $\phi_{(q-1) \lambda_{n}} \otimes \phi_{(q-1) \lambda_{n}}$ and $\left(\Pi_{n}\right)_{\operatorname{Sp}(2 m, q)}$ have the same multiplicities.

(2) The composition factors of $\left(V_{i}\right)_{\operatorname{Sp}(2 n, 2)}$ are also studied by Baranov and Suprunenko in 3 .

7.2.2. Indecomposable summands of $\sigma_{n} \otimes s t$. In this section we determine the indecomposable constituents of $\sigma_{n} \otimes s t$. Let $\nu$ be a dominant weight. We denote by $\phi_{\nu}$ the irreducible representation of $\mathbf{G}$ with highest weight $\nu$. Recall that every irreducible representation of $G=\operatorname{Sp}(2 n, q)$ is of shape $\left(\phi_{\nu}\right)_{G}$ where $\nu$ is a $q$-restricted dominant weight of $\mathbf{G}=\operatorname{Sp}(2 n, \mathbf{K})$. Put $\tilde{\omega}:=\lambda_{1}+\cdots+\lambda_{n}$. It is well known that $(q-1) \tilde{\omega}$ is the only $q$-restricted dominant weight $\rho$ such that $\left(\phi_{\rho}\right)_{G}=s t$. Recall that $\sigma_{n}=\left(\phi_{(q-1) \lambda_{n}}\right)_{G}$ and that $\phi_{(q-1) \lambda_{n}}$ is self-dual. 
Lemma $7.4([13,9.4])$. Let $\psi$ be an irreducible $\mathbb{F}_{q} G$-module. Then the multiplicity of the principal indecomposable module $\Phi_{\nu}$ in $\psi \otimes$ st is equal to the multiplicity of st in $\left(\phi_{\nu}\right)_{G} \otimes \psi^{*}$ where $\psi^{*}$ is the dual of $\psi$.

There is further information on those $\nu$ for which $\Phi_{\nu}$ may actually occur as a direct summand of $\psi \otimes s t$; see [13, 9.4]. We could prove Theorem [1.5] on the base of that information but our special case can probably be dealt with more efficiently staightforwardly. (Our argument here is based on Lemma 7.2 and general facts on representations of algebraic groups.)

Set $\nu=a_{1} \lambda_{1}+\cdots+a_{n} \lambda_{n}$ where $0 \leq a_{1}, \ldots, a_{n} \leq q-1$, and $\nu^{\prime}=a_{1} \lambda_{1}+\cdots+$ $a_{n-1} \lambda_{n-1}$.

Proof of Theorem 1.5. We show that $\Phi_{\nu}$ is a direct summand of $\sigma_{n} \otimes$ st if and only if $\nu^{\prime}=(q-1)\left(\lambda_{1}+\cdots+\lambda_{n-1}\right)$, that is, $a_{1}=\cdots=a_{n-1}=q-1$. It can be deduced from Steinberg [16, Corollary to Theorem 41 and Theorem 43] that $\phi_{\nu^{\prime}} \otimes \phi_{(q-1) \lambda_{n}}=\phi_{\nu^{\prime}+(q-1) \lambda_{n}}$. If $a_{n}=0$, we have $\nu=\nu^{\prime}$ so the representation $\phi_{\nu+(q-1) \lambda_{n}}$ is irreducible. As $\nu+(q-1) \lambda_{n}$ is a dominant $q$-restricted weight, $\left(\phi_{\nu+(q-1) \lambda_{n}}\right)_{G}$ is irreducible, so it is not equal to $s t$ unless $\nu=(q-1)\left(\lambda_{1}+\cdots+\lambda_{n-1}\right)$. So the claim follows from Lemma 7.4

Next assume $a_{n}>0$. Then we have that

$$
\phi_{\nu} \otimes \phi_{(q-1) \lambda_{n}}=\phi_{\nu^{\prime}} \otimes \phi_{a_{n} \lambda_{n}} \otimes \phi_{(q-1) \lambda_{n}} .
$$

Let $a_{n}=\sum_{i=0}^{k-1} 2^{i} b_{i}$ be the 2 -adic expansion of $a_{n}$ (so $0 \leq b_{i} \leq 1$ ). Then

$\phi_{a_{n} \lambda_{n}} \otimes \phi_{(q-1) \lambda_{n}}=\left(\phi_{b_{0} \lambda_{n}} \otimes \phi_{\lambda_{n}}\right) \otimes F_{0}\left(\phi_{b_{1} \lambda_{n}} \otimes \phi_{\lambda_{n}}\right) \otimes \cdots \otimes F_{0}^{k-1}\left(\phi_{b_{k-1} \lambda_{n}} \otimes \phi_{\lambda_{n}}\right)$. If $b_{i}=0$, then $\phi_{b_{i} \lambda_{n}} \otimes \phi_{\lambda_{n}}=\phi_{\lambda_{n}}$, otherwise $b_{i}=1$ and the composition factors of $\left(\phi_{b_{i} \lambda_{n}} \otimes \phi_{\lambda_{n}}\right)_{G}$ are $\left(\phi_{\lambda_{j}}\right)_{G}$ for $0 \leq j \leq n$ by Lemma 7.2. Therefore, the composition factors of $\left(\phi_{a_{n} \lambda_{n}} \otimes \phi_{(q-1) \lambda_{n}}\right)_{G}$ are the restrictions to $G$ of representations of shape

$$
\phi_{\lambda_{i_{0}}} \otimes F_{0}\left(\phi_{\lambda_{i_{1}}}\right) \otimes \cdots \otimes F_{0}^{k-1}\left(\phi_{\lambda_{i_{k-1}}}\right)=\phi_{\lambda_{i_{0}}+2 \lambda_{i_{1}}+\cdots+2^{k-1} \lambda_{i_{k-1}}}
$$

where $0 \leq i_{0}, i_{1}, \ldots, i_{k-1} \leq n$. Moreover, Lemma 7.2 tells us that the multiplicity of $\left(\phi_{\lambda_{j}}\right)_{G}$ in $\left(\phi_{b_{i} \lambda_{n}} \otimes \phi_{\lambda_{n}}\right)_{G}$ (when $\left.b_{i}=1\right)$ is at least 2 unless $j=n$. Therefore, every composition factor $\tau$, say, of

$$
\left(\phi_{\nu^{\prime}} \otimes \phi_{\lambda_{i_{0}}+2 \lambda_{i_{1}}+\cdots+2^{k-1} \lambda_{i_{k-1}}}\right)_{G}
$$

occurs at least twice unless $\lambda_{i_{0}}=\lambda_{i_{1}}=\cdots=\lambda_{i_{k-1}}=\lambda_{n}$ in which case $\lambda_{i_{0}}+2 \lambda_{i_{1}}+$ $\cdots+2^{k-1} \lambda_{i_{k-1}}=(q-1) \lambda_{n}$. It follows that $\tau \neq s t$ if $\tau$ occurs more than once, as otherwise, by Lemma 7.4, $\Phi_{\nu}$ occurs at least twice in $\left(\phi_{\nu} \otimes \phi_{(q-1) \lambda_{n}}\right)_{G}$ which contradicts Corollary 1.3

So we are left with determining the multiplicity of $s t$ in $\left(\phi_{\nu^{\prime}} \otimes \phi_{(q-1) \lambda_{n}}\right)_{G}$. As mentioned above, the latter representation coincides with $\left(\phi_{\nu^{\prime}+(q-1) \lambda_{n}}\right)_{G}$, which is irreducible. It coincides with st if and only if $\nu^{\prime}=(q-1)\left(\lambda_{1}+\cdots+\lambda_{n-1}\right)$.

Remark 7.5. The above reasoning also justifies the claim in Remark 7.3(1).

\section{ACKNOWLEDGEMENTS}

The second author greatfully acknowledges financial support by the DFG Research Training Group (Graduiertenkolleg) "Hierarchie und Symmetrie in mathematischen Modellen", and, at the final stage of the work, by a Leverhulme Emeritus Fellowship (Grant EM/2006/0030). 
A part of this work was done during a visit of the first author at the "Centre Interfacultaire Bernoulli" within the program "Group Representation Theory" (January to June 2005).

We thank Frank Lübeck for reassuring computations with CHEVIE 9 in an early state of this work, as well as for his careful reading of the manuscript. We also thank Frank Himstedt for his hint to reference [2]. Finally, we are indebted to Oliver Brunat for pointing out an inaccuracy in an earlier version of this article.

\section{REFERENCES}

[1] J. An and G. Hiss, Restricting the Steinberg character in finite symplectic groups, J. Group Theory 9 (2006), 251-264. MR2220418 (2007a:20011)

[2] J. An and S.-C. Huang, Character tables of parabolic subgroups of the Chevalley groups of type $G_{2}$, Comm. Algebra 34 (2006), 1763-1792. MR.2229489 (2007c:20014)

[3] A. A. Baranov and I. D. Suprunenko, Branching rules for modular fundamental representations of symplectic groups, Bull. London Math. Soc. 32 (2000), 409-420. MR1760805 (2001c:20099)

[4] N. Bourbaki, Groupes et algebres de Lie, Chaps. IV-VI, Hermann, Paris, 1968. MR 0240238 $(39: 1590)$

[5] J. Brundan, R. Dipper, and A. Kleshchev, Quantum linear groups and representations of $\mathrm{GL}_{n}\left(F_{q}\right)$, Mem. Amer. Math. Soc. 149 (2001), no. 706. MR.1804485 (2002h:20013)

[6] R. Carter, Finite groups of Lie type: Conjugacy classes and complex characters, Wiley, 1985. MR.794307 (87d:20060)

[7] F. Digne and J. Michel, Representations of finite groups of Lie type, London Math. Soc. Student Texts 21, Cambridge University Press, 1991. MR1118841 (92g:20063)

[8] K. Doerk and T. Hawkes, Finite soluble groups, De Gruyter, Berlin, 1992. MR.1169099 (93k:20033)

[9] M. Geck, G. Hiss, F. Lübeck, G. Malle, and G. Pfeiffer, CHEVIE-A system for computing and processing generic character tables, AAECC 7 (1996), 175-210. MR 1486215 (99m:20017)

[10] M. Geck and G. Pfeiffer, Characters of finite Coxeter groups and Iwahori-Hecke algebras, Oxford University Press, Oxford, 2000. MR 1778802 (2002k:20017)

[11] P. Gérardin, Weil representations associated to finite fields, J. Algebra 46 (1977), 54-101. MR0460477 (57:470)

[12] R. B. Howlett and G. I. Lehrer, Representations of generic algebras and finite groups of Lie type, Trans. Amer. Math. Soc. 280 (1983), 753-779. MR716849 (85i:20044)

[13] J. Humphreys, Modular representations of finite groups of Lie type, Cambridge University Press, Cambridge, 2006. MR 2199819 (2007f:20023)

[14] I. M. Isaacs, Characters of solvable and symplectic groups, Amer. J. Math. 95 (1973), 594635. MR0332945 (48:11270)

[15] G. Lusztig, On the representations of reductive groups with disconnected centre, Astérisque 168 (1988), 157-166. MR.1021495 (90j:20083)

[16] R. Steinberg, Lectures on Chevalley Groups, mimeographed lecture notes, Yale Univ. Math. Dept., New Haven, Conn., 1968. MR0466335 (57:6215)

[17] D. A. Suprunenko, Soluble and nilpotent linear groups (In Russian), Belorussian Univ. Press, Minsk, 1958 (English translation: Amer. Math. Soc. Providence, 1963). MR0154918 $(27: 4861 b)$

[18] I. D. Suprunenko and A. E. Zalesskii, Permutation representations and a fragment of the decomposition matrix of the symplectic and the special linear groups over a finite field, Siber. Math. J. 31 (1990), 744-755. MR1088915 (92a:20053)

[19] A. E. Zalesskii, A fragment of the decomposition matrix of the special unitary group over a finite field, Math. USSR, Izvestija 36 (1991), 23-39. MR.1044046 (91d:20012) 


\title{
Appendix. Restricting the Steinberg Character IN FINITE LINEAR AND UNITARY GROUPS
}

by Olivier Brunat

\begin{abstract}
Let $G$ be one of the groups $\operatorname{GL}(n, q)$ or $U(n, q)$, and let $H$ denote the subgroup $\operatorname{GL}(n-1, q)$ or $U(n-1, q)$, respectively. We show that the restriction of the Steinberg character of $G$ to $H$ equals the product of the Weil character and the Steinberg character of $H$.
\end{abstract}

For the convenience of the reader, we recall some notations: Let $\mathbf{G}=\operatorname{GL}\left(n, \overline{\mathbb{F}}_{p}\right)$ and let $F$ be the Frobenius endomorphism which acts by raising all entries of a matrix to the $q$ th power, where $q$ is a power of $p$. We set $\sigma:\left(a_{i j}\right) \mapsto\left(\left(a_{i j}\right)^{-1}\right)^{t}$ and $F^{\prime}=F \circ \sigma$. The fixed-point subgroups $\mathbf{G}^{F}$ and $\mathbf{G}^{F^{\prime}}$ are the finite linear group $\operatorname{GL}(n, q)$ and the unitary group $U(n, q)$, respectively. We set

$$
\Phi: \mathrm{GL}\left(n-1, \overline{\mathbb{F}}_{p}\right) \rightarrow \mathrm{GL}\left(n, \overline{\mathbb{F}}_{p}\right), \quad x \mapsto\left[\begin{array}{ll}
x & 0 \\
0 & 1
\end{array}\right] .
$$

We have $\Phi(x)^{F}=\Phi\left(x^{F}\right)$ and $\Phi(x)^{F^{\prime}}=\Phi\left(x^{F^{\prime}}\right)$; in the right-hand side of the equalities, $F$ (respectively $F^{\prime}$ ) denotes the map defined previously, but for $\operatorname{GL}\left(n-1, \overline{\mathbb{F}}_{p}\right)$. Using $\Phi$, we embed $\operatorname{GL}(n-1, q)$ in $\operatorname{GL}(n, q)$ and $U(n-1, q)$ in $U(n, q)$. We identify $\operatorname{GL}(n-1, q)$ with $\mathbf{H}^{F}$ and $U(n-1, q)$ with $\mathbf{H}^{F^{\prime}}$, where $\mathbf{H}=\Phi\left(\operatorname{GL}\left(n-1, \overline{\mathbb{F}}_{p}\right)\right)$. We denote by $\mathbf{S t}_{\mathbf{G}}$ and $\mathbf{S t}_{\mathbf{H}}$ the Steinberg characters of these groups. Note that these characters depend on the Frobenius maps $F$ and $F^{\prime}$. We set $\left.\operatorname{St}_{r}=\operatorname{Res}_{\mathbf{H}^{F}} \mathbf{S}_{\mathbf{G}}\right)$ and we use the same symbol for the restriction of $\mathrm{St}_{\mathbf{G}}$ from $\mathbf{G}^{F^{\prime}}$ to $\mathbf{H}^{F^{\prime}}$. Finally, we denote by $\omega_{n}$ the Weil character of $\operatorname{GL}(n, q)$ and that of $U(n, q)$, in each case using the version of Gérardin [2].

Lemma. With the preceding notation we have

$$
\mathrm{St}_{r}=\omega_{n-1} \otimes \mathrm{St}_{\mathbf{H}} .
$$

Proof. Let $\widetilde{F}$ denote $F$ or $F^{\prime}$. We denote by $\mathcal{S}$ the set of semisimple elements of $\mathbf{H}^{\widetilde{F}}$. Following [1, 6.5.9], if $g \notin \mathcal{S}$, we have $\operatorname{St}_{r}(g)=0=\omega_{n-1} \otimes \operatorname{St}_{\mathbf{H}}(g)$. Moreover, if $s \in \mathcal{S}$, then

$$
\operatorname{St}_{r}(s)=\varepsilon_{\mathbf{G}} \varepsilon_{\mathrm{C}_{\mathbf{G}}(s)}\left|\mathrm{C}_{\mathbf{G}}(s)^{\widetilde{F}}\right|_{p} \quad \text { and } \quad \operatorname{St}_{\mathbf{H}}(s)=\varepsilon_{\mathbf{H}} \varepsilon_{\mathrm{C}_{\mathbf{H}}(s)}\left|\mathrm{C}_{\mathbf{H}}(s)^{\widetilde{F}}\right|_{p} .
$$

Fix $s \in \mathcal{S}$. Then there is $t \in \mathbf{H}^{\widetilde{F}}$ such that

$$
{ }^{t} s=\left[\begin{array}{llll}
A & & & \\
& 1 & & \\
& & \ddots & \\
& & & 1
\end{array}\right],
$$

and 1 is not an eigenvalue of $A$. Let $i$ be the multiplicity of the eigenvalue 1 of $s$, thus $\mathrm{C}_{\mathbf{H}}\left({ }^{t} s\right)=\mathrm{C}_{\mathbf{H}}(A) \times \mathrm{GL}\left(i-1, \overline{\mathbb{F}}_{p}\right)$. Moreover, we have $\mathrm{C}_{\mathbf{G}}\left({ }^{t} s\right)=\mathrm{C}_{\mathbf{G}}(A) \times$ $\mathrm{GL}\left(i, \overline{\mathbb{F}}_{p}\right)$. But $\mathrm{C}_{\mathbf{G}}(A)=\mathrm{C}_{\mathbf{H}}(A)$, and so $\mathrm{C}_{\mathbf{G}}\left({ }^{t} s\right)=\mathrm{C}_{\mathbf{H}}(A) \times \mathrm{GL}\left(i, \overline{\mathbb{F}}_{p}\right)$. It follows that

$$
\begin{aligned}
\left|\mathrm{C}_{\mathbf{G}}\left({ }^{t} s\right)^{\widetilde{F}}\right|_{p} & =\left|\mathrm{C}_{\mathbf{H}}(A)^{\widetilde{F}}\right|_{p}\left|\operatorname{GL}\left(i, \overline{\mathbb{F}}_{p}\right)^{\widetilde{F}}\right|_{p} \\
& =\left|\mathrm{C}_{\mathbf{H}}\left({ }^{t} s\right)^{\widetilde{F}}\right|_{p}\left|\operatorname{GL}\left(i, \bar{F}_{p}\right)^{\widetilde{F}}\right|_{p} /\left|\operatorname{GL}\left(i-1, \overline{\mathbb{F}}_{p}\right)^{\widetilde{F}}\right|_{p} \\
& =q^{i-1}\left|\mathrm{C}_{\mathbf{H}}\left({ }^{t} s\right)^{\widetilde{F}}\right|_{p} .
\end{aligned}
$$


Moreover, we have

$$
\begin{aligned}
\operatorname{rk}_{\mathbb{F}_{q}}\left(\mathrm{C}_{\mathbf{G}}\left({ }^{t} s\right)\right) & =\operatorname{rk}_{\mathbb{F}_{q}}\left(\mathrm{C}_{\mathbf{H}}(A)\right)+\operatorname{rk}_{\mathbb{F}_{q}}\left(\mathrm{GL}\left(i, \overline{\mathbb{F}}_{p}\right)\right), \\
& =\operatorname{rk}_{\mathbb{F}_{q}}\left(\mathrm{C}_{\mathbf{H}}\left({ }^{t} s\right)\right)+\operatorname{rk}_{\mathbb{F}_{q}}\left(\operatorname{GL}\left(i, \overline{\mathbb{F}}_{p}\right)\right)-\operatorname{rk}_{\mathbb{F}_{q}}\left(\mathrm{GL}\left(i-1, \overline{\mathbb{F}}_{p}\right)\right) .
\end{aligned}
$$

Suppose that $\widetilde{F}=F$. We then have $\operatorname{rk}_{\mathbb{F}_{q}}\left(\operatorname{GL}\left(i, \overline{\mathbb{F}}_{p}\right)\right)-\operatorname{rk}_{\mathbb{F}_{q}}\left(\operatorname{GL}\left(i-1, \overline{\mathbb{F}}_{p}\right)\right)=1$, and it follows that

$$
\varepsilon_{\mathrm{C}_{\mathrm{G}}(s)} \varepsilon_{\mathrm{C}_{\mathbf{H}}(s)}=-1 .
$$

In particular, we have $\varepsilon_{\mathbf{G}} \varepsilon_{\mathbf{H}}=-1$ and we deduce that

$$
\begin{aligned}
\mathrm{St}_{r}(s) & =\varepsilon_{\mathbf{G}} \varepsilon_{\mathrm{C}_{\mathbf{G}}(s)} q^{i-1}\left|\mathrm{C}_{\mathbf{H}}\left({ }^{t} s\right)^{F}\right|_{p} \\
& =\varepsilon_{\mathbf{G}} \varepsilon_{\mathrm{C}_{\mathbf{G}}(s)} q^{i-1} \varepsilon_{\mathbf{H}} \varepsilon_{\mathrm{C}_{\mathbf{H}}\left({ }^{t} s\right)} \operatorname{St}_{\mathbf{H}}(s) \\
& =q^{i-1} \operatorname{St}_{\mathbf{H}}(s) .
\end{aligned}
$$

Following 2, we have $\omega_{n-1}(s)=q^{i-1}$ proving the claim in this case.

Suppose that $\widetilde{F}=F^{\prime}$. Then

$$
\operatorname{rk}_{\mathbb{F}_{q}}\left(\operatorname{GL}\left(i, \overline{\mathbb{F}}_{p}\right)\right)-\operatorname{rk}_{\mathbb{F}_{q}}\left(\operatorname{GL}\left(i-1, \overline{\mathbb{F}}_{p}\right)\right)=\lfloor i / 2\rfloor-\lfloor(i-1) / 2\rfloor .
$$

Hence, we have

$$
\varepsilon_{\mathrm{C}_{\mathbf{G}}(s)} \varepsilon_{\mathrm{C}_{\mathbf{H}}(s)}=(-1)^{\lfloor i / 2\rfloor-\lfloor(i-1) / 2\rfloor}=(-1)^{i-1} .
$$

In particular, we have $\varepsilon_{\mathbf{G}} \varepsilon_{\mathbf{H}}=(-1)^{n-1}$. As before, we deduce that

$$
\mathrm{St}_{r}(s)=(-1)^{n-1}(-q)^{i-1} \operatorname{St}_{\mathbf{H}}(s) \text {. }
$$

Moreover, since $\omega_{n-1}(s)=(-1)^{n-1}(-q)^{i-1}$, the claim follows.

\section{REFERENCES}

[1] R. Carter, Finite groups of Lie type: Conjugacy classes and complex characters, Wiley, 1985. MR794307 (87d:20060)

[2] P. Gérardin, Weil representations associated to finite fields, J. Algebra 46 (1977), 54-101. MR0460477 (57:470)

Lehrstuhl D für Mathematik, RWTH Aachen University, 52056 Aachen, Germany

E-mail address: gerhard.hiss@math.rwth-aachen.de

School of Mathematics, University of East Anglia, Norwich, NR47TJ, United KingDOM

E-mail address: alexandre.zalesski@gmail.com

FAKUltät Für Mathematik, RUhr-Universität Bochum, Universitätsstrasse 150, 44780 Bochum

E-mail address: olivier.brunat@ruhr-uni-bochum.de 